\title{
Updated Checklist of Food Plants of Macrosiphini (Aphididae: Hemiptera) in India - 1
}

\author{
${ }^{1}$ Garima Singh, ${ }^{2}$ Rajendra Singh \\ ${ }^{1}$ Department of Zoology, Rajasthan University, Jaipur, Rajasthan. \\ ${ }^{2}$ Department of Zoology, D.D.U. Gorakhpur University, Gorakhpur, U.P.
}

\begin{abstract}
The Macrosiphini is one of the 2 tribes of the subfamily Aphidinae (Aphididae: Hemiptera) containing about 2166_species/subspecies assigned to 242 genera. Out of these, only 96 genera and 374 species are recorded from India infesting hundreds of plant species belonging to several families. The food plants of Indian Macrosiphini is catalogued alphabetically (aphid species-wise) in three parts. In this contribution, food plants of only 25 genera and 109 species of aphids belonging to the tribe Macrosiphini (Acutosiphon to Dysaphis) are catalogued. These aphids feeds on 400 plant species belonging to 79 families. Most suffered families are Asteraceae (81 species) followed by Rosaceae (35 species), Fabaceae (27 species), Brassicaceae (22 species), Lamiaceae (18 species) and Solanaceae (17 species). Among them, several species are crops of agricultural and horticultural importance. The update checklist of food plants of rest of Macrosiphini will be dealt in successive articles.
\end{abstract}

Keywords: Food plant, Macrosiphini, aphid, checklist.

\section{INTRODUCTION}

Aphids are a group of over 5000 species of small plant sap sucking insects, popularly known as plantlice or ant-cows and vary in size between 0.7 to $7.0 \mathrm{~mm}$ in length. Many species of aphids are Agricultural and horticultural crop pests. In India, Raychaudhuri [1] for the first time catalogued the food-plants of Indian Aphididae, later on, Chakrabarti and Sarkar [2] added additional records. Since then, there are several reports that deal with the food plant records of the Indian aphids. Recently, Singh and Singh [3-6] and Tiwari \& Singh [7] have initiated to compile the food plant records of the aphids in India. This series of publication is a comprehensive, systematic, up-to-date catalogue of the recorded aphid species of India, their recorded host plants and relevant literature. The present paper deals with the food plant records of Indian aphids belonging to tribe Macrosiphini (Aphidinae: Aphididae: Hemiptera) based on the survey of literature up to 1 January, 2017. In the most of the literature published earlier, several errors crept in scientific names of the aphids and plants even in the recent ones. It happens because such contents become outdated quickly and, due to their perceived comprehensiveness, readers sometimes overlook newer sources of data. Additionally, the researches on aphid taxonomy as well as their host plants is continued with the description of new taxa, the modified status of others, and the publication of other nomenclatural decisions. In the present compilation, attempts have been made to correct these errors in the scientific names of the aphids following recent taxonomic literatures [8-11] and for plants, http://www.ars-grin.gov and $\mathrm{http}: / / \mathrm{www}$.theplantlist. org (version 1.1). At several places, their synonymies were also mentioned.

The tribe Macrosiphini Wilson, 1910 is one of the two tribes (Aphidini and Macrosiphini) of the subfamily Aphidinae (Aphididae: Hemiptera) which is the largest subfamily of the aphids containing 2166 species assigned to 242 genera [11]. The Macrosiphini can be differentiated by Aphidini by the following morphological characters: spiracles of abdominal segments 1 and 2 are placed closed, lateral abdominal tubercles are usually absent from segments 1 and 7 but variably present on segments 2-3 or completely absent, and the antennal tubercles are often well developed. Several members of the tribe are major agricultural pests damaging the crops directly by sucking their sap or by transmitting viral diseases. Myzus persicae (Sulzer) alone transmit about 180 plant viruses [7, 12]. The food plants of Aphidini was recently catalogued [6]. 


\section{APHID - FoOd Plants ReCORD OF MACROSIPHINI}

As mentioned earlier, the tribe Macrosiphini is largest tribe of the family Aphididae containing more than two thousand species, the checklist of food plants are being published in 3 parts and this one is the first in series. Aphid species marked with $\S$ seems to be invalid while species marked with * are wrongly reported for another valid species. Following is their food plant record:

\section{Acutosiphon obliquoris Basu, Ghosh \& Raychaudhuri, 1970}

- Carex cespitosa L. (=Carex arex auct. nonn. ? Carex ferax Raeuch.) (Cyperaceae) [13]

- Carex filicina Nees (=Carex filicosa auct. nonn.) (Cyperaceae) [14-20]

- Carex sp. (Cyperaceae) $[18,19,21]$

- Cotula sp. (Asteraceae) [18-20, 22]

- Cyperus sp. (Cyperaceae) [17, 23]

- Fragaria nilgerrensis Schltdl. ex J. Gay (Rosaceae) [14, 15]

- Geranium sp. (Geraniaceae) $[14,15]$

- Poa sp. (Poaceae) [24]

- Undet.: Cyperaceae [1]; Poaceae) [14]

2. Acyrthosiphon (Acyrthosiphon) ? caraganae (Cholodkovsky, 1907)

$=$ Acyrthosiphon $\mathrm{nr}$. caraganae (Cholodkovsky, 1907) [19, 25]

= Metopolophium (Metopolophium) ? caraganae (Cholodkovsky, 1907) [1]

- Cajanus cajan (L.) Millsp. (Fabaceae) [19, 20, 26]

- Lablab purpureus (L.) Sweet (=Dolichos lablab L.) (Fabaceae) [19, 25]

3. Acyrthosiphon (Acyrthosiphon) gossypii Mordvilko, 1914

= Acyrthosiphon sesbaniae David, 1956 [27]

= Macrosiphum (Acyrthosiphon) gossypii Mordvilko [27]

- Sesbania grandiflora (L.) Pers. (Fabaceae) [28, 29]

- Sophora tomentosa L. (Fabaceae) [27]

4. Acyrthosiphon (Acyrthosiphon) ignotum Mordvilko, 1914

= Acyrthosiphon (Metopolophium) spireae Ghosh, Chakrabarti, Chowdhuri \& Rauchaudhuri, 1969

= Acyrthosiphon (Metopolophium) spireae canescentis Chakrabarti \& Rauchaudhuri, 1974 [17]

- Dactylus sp. (Poaceae) [17, 30]

- Deutzia corymbosa Craib. (Hydrangeaceae) [31]

- Spiraea canescens D. Don (Rosaceae) [17, 30]

5. Acyrthosiphon (Acyrthosiphon) kondoi Shinji, 1938

- Medicago sativa L. (Fabaceae) [32]

6. Acyrthosiphon (Acyrthosiphon) malvae (Mosley, 1841)

= Acyrthosiphon pelargonii (Kaltenbach, 1843) [24, 33, 34]

= Acyrthosiphon pelargonii geranii (Kaltenbach, 1843) [19]

= Metopolophium (Metopolophium) malvae complex [1]

- Anemone rivularis Buch.-Ham. ex DC. (Ranunculaceae) [19]

- Calceolaria sp. (Scrophulariaceae) [35, 36]

- Geranium sp. (Geraniaceae) [19, 20, 26]

- Potentilla sp. (Rosaceae) [14, 19]

- Rosa sp. (Rosaceae) [19]

- Rubus ellipticus Sm. (Rosaceae) [31]

- Salvia cuspidata subsp. gilliesii (Benth.) J.R.I.Wood (=Salvia glandulosa, probably Salvia gilliesii var. glandulosa Griseb.) (Lamiaceae) $[17,37]$

- Senecio sp. (Asteraceae) [34]

- Sisymbrium sp. (Brassicaceae) [14, 19, 33]

- Undet.: Acanthaceae [19]; Fabaceae [38]

7. Acyrthosiphon (Acyrthosiphon) moltshanovi Mordvilko, 1914

- Lactuca sativa L. (Asteraceae) [39, 40] 
8. Acyrthosiphon (Acyrthosiphon)? moltshanovi Mordvilko, 1914

- Lactuca sativa L. (Asteraceae) [17]

- Peucedanum tenuifolium Thunb. (Apiaceae) [17,41]

9. Acyrthosiphon (Acyrthosiphon) phaseoli Chakrabarti, Ghosh \& Raychaudhuri, 1971

- Phaseolus sp. (Fabaceae) [17, 39, 42]

10. Acyrthosiphon (Acyrthosiphon) pisum (Harris, 1776)

= Illinoia pisi Kaltenbach [43]

= Macrosiphum pisi Kaltenbach [44-46]

- Alhagi maurorum Medik. (=Alhagi camelorum Fisch.) (Fabaceae) $[28,47]$

- Cajanus cajan (L.) Millsp. (Fabaceae) [48]

- Chenopodium album L. (Chenopodiaceae) [14, 49]

- Cicer arietinum L. (Fabaceae) $[39,50]$

- Dianthus dampieri (Caryophyliaceae, misident. ?) [28, 47]

- Glycine max (L.) Merr. (Fabaceae) [14]

- Lablab purpureus (L.) Sweet (=Dolichos lablab L.) (Fabaceae) $[28,47]$

- Lathyrus odoratus L. (Fabaceae) [28, 47, 51-54]

- Lathyrus sativus L. (Fabaceae) [50, 52, 53, 55]

- Launaea nudicaulis (Linn.) Hook.f. (Asteraceae)801

- Lens culinaris Medik. (Fabaceae) [52, 53]

- Lens culinaris Medik (=Lens esculenta Moench.) (Fabaceae) [55]

- Medicago falcata L. (=Medicago falcatum) (Fabaceae) [28]

- Medicago lupulina L. (Fabaceae) [28, 45, 47]

- Medicago polymorpha L. (=Medicago denticulata Willd.) (Fabaceae) [28, 45, 47]

- Medicago sativa L. (Fabaceae) [28, 45, 47, 50, 53]

- Melilotus albus Medik (Fabaceae) $[28,47]$

- Peganum hirmala L. (Nitrariaceae) $[28,47]$

- Pisum sativum L. (Fabaceae) [14, 26, 28, 39, 43, 47, 49, 56-60]

- Pisum sp. (Fabaceae) [19, 20, 26]

- Prinsepia utilis Royle (Rosaceae) [14, 19]

- Pyrus communis L. (Rosaceae) [19]

- Sesbania grandiflora (L.) Pers. (Fabaceae) $[28,43]$

- Solanum tuberosum L. (Solanaceae) $[19,47]$

- Trifolium alexandrinum L. (=Trigonella alexandrium, misident. ?) (Fabaceae) [50]

- Trigonella foenum-graecum L. (Fabaceae) [28, 50, 53]

- Vicia faba L. (Fabaceae) [19, 20, 26, 50, 52, 53]

11. Acyrthosiphon (Acyrthosiphon) ranunculum Ghosh, 1986

= Acyrthosiphon (Metopolophium) ranunculum Ghosh, 1986)

- Thalictrum pauciflorum Royle (Ranunculaceae) [37]

12. Acyrthosiphon (Acyrthosiphon) rubi (Narzikulov, 1957)

= Acyrthosiphon rubi elliptici Stroyan \& Nagaich, 1964 [14, 36, 61, 62]

= Acyrthosiphon (Metopolophium) rubi Narzikulov, 1957 [14, 17, 41, 63, 64, 65, 66]

= Acyrthosiphon rubi (Narzikulov, 1957) [17, 19, 23, 63]

= Metopolophium (Metopolophium) rubi (Narzikulov, 1957) [1]

= Metopolophium sonchifoliae Raychaudhuri, Ghosh \& Das, 1980

- Catamixis baccaroides Thomson (Asteraceae) [17]

- Desmodium sp. (Fabaceae) [67]

- Fagopyrum esculentum Moench (Polygonaceae) [41, 68]

- Fagopyrum tataricum (L.) Gaertn. (Polygonaceae) [17, 41]

- Hieracium lachenalii subsp. cruentifolium (Dahlst. \& Lübeck) Zahn (=Hieracium vulgatum Fr.) (Asteraceae) [17, 41]

- Polygonum sp. (Polygonaceae) [67]

International J. Research Studies in Zoology (IJRSZ) 
- Rhododendron sp. (Ericaceae) [17]

- Rubus croceacanthus H. Lév (=Rubus asper Wall. ex D. Don) (Rosaceae) [14, 19]

- Rubus ellipticus Sm. (Rosaceae) [17, 19, 20, 24, 26, 67, 69, 70]

- Rubus fruticosus Lucviatour. JPG (Rosaceae) [71]

- Rubus niveus Thunb. (Rosaceae) [14]

- Rubus plicatus Weihe \& Nees (=Rubus fruticosus L.) (Rosaceae) [72]

- Rubus sp. (Rosaceae) [14, 17, 23, 26, 41, 63, 67]

- Rubus ulmifolius Schott (Rosaceae) [31]

- Rumex nepalensis Spreng. (Polygonaceae) [14, 64, 73]

- Rumex sp. (Polygonaceae) [17, 19, 20, 26, 41]

- Saussurea costus (Falc.) Lipsch. (=Saussurea lappa (Dacne.) C.B. Clarke) (Asteraceae) [17, 41]

- Senecio sp. (Asteraceae) [34]

- Sonchus oleraceus L. (Asteraceae) [14]

- Sonchus sp. (Asteraceae) [68]

- Undet.: Rosaceae $[20,26,41,68]$

13. Acyrthosiphon (Acyrthosiphon) rubifoliae Raychaudhuri, Ghosh \& Basu, 1975 (1978)

= Metopolophium (Microlophium) rubifoliae Raychaudhuri, Ghosh \& Basu, 1975 (1978) [1]

- Potentilla nepalensis Hook (Rosaceae) [68]

- Rubus ellipticus Sm. (Rosaceae)1, 19, 20, 26, 79, 106]

- Rubus plicatus Weihe \& Nees (=Rubus fruticosus L.) (Rosaceae) [55]

- Rubus rosifolius $\mathrm{Sm}$. (=Rubus rosaefolia auct.) (Rosaceae) [19, 25]

- Rubus sp. (Rosaceae) [19]

14. Acyrthosiphon (Acyrthosiphon) ? soldatovi Mordvilko, 1914

- Undet.: [17]

15. Acyrthosiphon (Acyrthosiphon) sp.

- Bothriochloa sp. (Poaceae) [74]

- Catamixis sp. (Asteraceae) [39]

- Euphorbia pilosa L. (Euphorbiaceae) [75]

- Hieracium sp. (Asteraceae) [39]

- Medicago sativa L. (Fabaceae ) [72]

- Ranunculus muricatus L. (Ranunculaceae) [72]

- Salix elaeagnos Scop. (=Salix elegans auct. non.) (Salicaceae) [76]

16. Acyrthosiphon calatropi Sathe \& Jadhav, 2008§

- Calotropis gigantea (L.) W.T. Aiton (Apocynaceae) [77]

17. Acyrthosiphon vatanii Sathe \& Jadhav, 2008§

- Pisum sativum Linn. (Fabaceae) [77]

18. Akkaia bengalensis Basu, 1968

- Koenigia mollis (D. Don) T. M. Schust. \& Reveal (=Polygonum molle D. Don.) (Polygonaceae) $[19,78]$

- Persicaria barbata (L.) H. Hara (=Polygonum barbatum L.) (Polygonaceae) $[19,78,79]$

- Persicaria glabra (Willd.) M. Gomez (=Polygonum glabrum Willd.) (Polygonaceae) [19, 78]

- Persicaria perfoliata (L.) H. Gross (=Polygonum perfoliatum L.) (Polygonaceae) [19]

- Polygonum alatum Buch.-Ham. ex D. Don (Polygonaceae) [19, 78, 79]

- Polygonum microcephalum var. sphaerocephalum (Wall. ex Meisner.) (=Polygonum sphaerocephalum Wall. ex Meisner.) (Polygonaceae) [80]

- Polygonum runcinatum Buch.-Ham. ex D. Don (Polygonaceae) [19]

- Polygonum sp. (Polygonaceae) [18-20, 78, 81]

- Raphanus sativus L. (Brassicaceae) [81]

- Rumex sp. (Polygonaceae) $[18,19]$ 
19. Akkaia neopolygoni Ghosh, Ghosh \& Raychaudhuri, 1970

- Koenigia mollis (D. Don) T. M. Schust. \& Reveal) (=Polygonum molle D. Don.) (Polygonaceae) $[19,78,79]$

- Persicaria decipiens (R.Br.) K.L.Wilson (=Polygonum serrulatum Lagasca) (Polygonaceae) [19, 82]

- Polygonum sp. (Polygonaceae) [18-20, 25, 82]

20. Akkaia sikkimensis Agarwala \& Raychaudhuri, 1977

- Raphanus sativus L. (Brassicaceae) [78, 83]

21. Amphicercidus japonicus (Hori, 1927)

=Amphicercidus indicus Hille Ris Lambers \& Basu, 1966

- Lonicera glabrata Wall. (Caprifoliaceae) $[20,84]$

22. Amphicercidus lonicerae Maity \& Chakrabarti, 1982

- Lonicera quinquelocularis Hardw. (Caprifoliaceae) [31]

- Lonicera sp. (Caprifoliaceae) [85]

23. Amphicercidus tuberculatus David, Narayanan \& Rajasingh, 1970

- Buxus wallichiana Baill. (Buxaceae) [17]

- Lonicera quinquelocularis Hardw. (Caprifoliaceae) [17, 86, 87]

- Lonicera sp. (Caprifoliaceae) [88]

24. Amphorophora (Amphorophora) ampullata ampullata Buckton, 1876

- Asplenium sp. (Aspleniaceae) [89]

25. Amphorophora (Amphorophora) ampullata bengalensis Hille Ris Lambers \& Basu, 1966

- Asplenium sp. (Aspleniaceae) [17, 19, 20]

- Athyrium sp. (Dryopteridaceae) $[19,20]$

- Cheilanthes sp. (Pteridaceae) $[19,20,89,90]$

- Eriosorus sp. (Pteridaceae) [78]

- Gleichenia sp. (Gleicheniaceae) [19]

- Hypolepis polypodioides (Blume) Hook. (Hypolepidaceae) [91]

- Polypodium sp. (Polypodiaceae) [19, 20]

- Pteridium aquilinum (L.) Kulm (=Pteris aquilina L.) (Pteridaceae) $[19,20,89,90]$

- Pteris sp. (Pteridaceae) [78]

- Rosa sp. (Rosaceae) [78]

26. Amphorophora (Amphorophora) sp.

- Rubus assamensis Focke (Rosaceae) [50]

- Rubus ellipticus Sm. (Rosaceae) [50]

- Rubus moluccanus L. (Rosaceae) [50, 92]

27. Anaulacorthum fagopyri (Ghosh \& Raychaudhuri, 1972)

= Aulacorthum (Anaulacorthum) fagopyri Ghosh \& Raychaudhuri, 1972

- Fagopyrum acutatum (Lehm.) Mansf. ex K. Hammer (=Fagopyrum cymosum (Trev.) Meisn) (Polygonaceae) [20, 93]

- Persicaria perfoliata (L.) H. Gross (=Polygonum perfoliatum L.) (Salicaceae) [20, 55]

28. Aphidura ornatella Narzikulov \& Umarov, 1969

= Aphidura bharatia David, Sekhon \& Bindra, 1970

- Saponaria sp. (Caryophyliaceae) [94]

29. Aphidura sp.

- Saponaria sp. (Caryophyliaceae) [95]

International J. Research Studies in Zoology (IJRSZ)

Page $\mid 10$ 
30. Aspidophorodon (Aspidophorodon) harvensis Verma, 1966

- Populus ciliata Wall. ex Royle (Saliaceae) [96]

- Salix sp. (Salicaceae) [96-98]

31. Aspidophorodon (Eoessigia) indica (David, Rajasingh \& Narayanan, 1972)

= Eoessigia indica David, Rajasingh \& Narayanan, 1972

= Raychaudhuriella myzaphoides Chakrabarti, 1978

= Raychaudhuriella potentillae Chakrabarti \& Maity, 1984

- Cotoneaster sp. (Rosaceae) [17, 37, 86]

- Cotoneaster bacillaris Wall. ex Lindl. (=Cotoneaster obtusus Wall. ex Lindl.) (Rosaceae) [99]

- Potentilla sp. (Rosaceae) [99, 100]

32. Aspidophorodon (Eoessigia) sorbi (Chakrabarti \& Maity, 1984)

= Indotuberoaphis sorbi Chakrabarti \& Maity, 1984

- Sorbus foliolosa (Wall.) Spach (Rosaceae) [100]

33. Aulacorthum (Aulacorthum) cornaceae Ghosh, 1969

- Undet.: Cornaceae [20]

34. Aulacorthum (Aulacorthum) dasi Ghosh, Basu \& Raychaudhuri, 1970

- Artemisia sp. (Asteraceae) [19]

- Solanum torvum Sw. (Solanaceae) [19, 20, 78]

- Undet.: Asteraceae) $[20,101]$

35. Aulacorthum (Aulacorthum) linderae (Shinji, 1922)

- Cucurbita moschata Duchesne (Cucurbitaceae) [102]

36. Aulacorthum (Aulacorthum) rhamni Ghosh, Ghosh \& Raychaudhuri, 1970 (1971)

- Callistemon linearis (Schrad. \& Wendl.) Colv. ex Sweet (Myrtaceae) [19]

- Duranta sp. (Verbenaceae) [2]

- Eurya sp. (Ternstroemiaceae) $[19,20]$

- Rhamnus napalensis (Wall.) M.A. Lawson (Rhamnaceae) [19, 20, 103]

- Saruma sp. (Aristolochiaceae) [20, 78]

- Undet.: Euphorbiaceae [20]

37. Aulacorthum (Aulacorthum) sclerodorsi (Kumar \& Burkhandt, 1971)

= Acyrthosiphon (Aulacorthum) sclerodorsi Kumar \& Burkhandt, 1971 [17]

- Nepeta sp. (Lamiaceae) [17, 104]

38. Aulacorthum (Aulacorthum) sensoriatum (David, Narayanan \& Rajasingh, 1970 (1971))

= Acyrthosiphon (Aulacorthum) sensoriatum David, Narayanan \& Rajasingh, 1970 (1971)

- Undet.: [17]

39. Aulacorthum (Aulacorthum) solani (Kaltenbach, 1843)

= Acyrthosiphon (Aulacorthum) solani (Kaltenbach, 1843) : David \& Rajasingh, 1969

- Ageratum conyzoides L. (Asteraceae) [19]

- Bidens sp. (Asteraceae) [67]

- Brassica oleracea L. (Brassicaceae) [19]

- Calla sp. (Araceae) [81]

- Cardamine hirsuta L. (Brassicaceae) [17]

- Cestrum fasciculatum (Schltdl.) Miers (Solanaceae) [19]

- Chrysanthemum sp. (Asteraceae) [67]

- Cineraria sp. (Asteraceae) [47]

- Clinopodium umbrosum (M. Bieb.) K. Kuntze (=Calamintha umbrosa (M. Bieb.) Fisch. \& C. A. Mey.) (Lamiaceae) $[14,105]$ 
- Consularia sp. (Asteraceae) [67]

- Datura sp. (Solanaceae) [20]

- Dianthus sp. (Caryophyliaceae) [106]

- Digitalis purpurea L. (Plantaginaceae) $[28,47]$

- Drymaria cordata (L.) Willd. ex Schult. (Caryophyliaceae) [19]

- Fagopyrum acutatum (Lehm.) Mansf. ex K.Hammer (=Fagopyrum cymosum (Trev.) Meisn) (Polygonaceae) [19, 20]

- Gerbera sp. (Asteraceae) [16]

- Gladiolus sp. (Iridaceae) [67]

- Hibiscus rosa-sinensis L. (Malvaceae) [55, 101, 106]

- Hydrangea sp. (Hydrangeaceae) [31]

- Lobelia sp. (Campanulaceae) [81]

- Nepeta sp. (Lamiaceae) [2]

- Nicotiana tabacum L. (Solanaceae) [19, 20]

- Oxalis sp. (Oxalidaceae) [107]

- Paphiopedilum venustum (Wall. ex Sim.) Pftizer (Orchidaceae) [20, 101]

- Poa sp. (Poaceae) [107]

- Pseudognaphalium luteoalbum (L.) Hill. \& Burtt (=Gnaphalium luteoalbum L.) (Asteraceae) [20]

- Ranunculus arvensis L. (Ranunculaceae) [14,19, 105]

- Rosa sp. (Rosaceae) [17]

- Salvia sp. (Lamiaceae) [17]

- Sechium edule (Jacq.) Sw. (Cucurbitaceae) [108]

- Solanum donianum Walp. (=Solanum verbascifolium L.) (Solanaceae) [81]

- Solanum melongena L. (Solanaceae) [108]

- Solanum sp. (Solanaceae) [20]

- Solanum tuberosum L. (Solanaceae) [19, 20, 47, 81, 107]

- Tagetes patula L. (Asteraceae) [106, 108]

- Viola sp. (Violaceae) [14, 17, 19, 39]

- Xerochrysum bracteatum (Vent.) Tzvelev (=Helichrysum bracteatum (Vent.) Andrews) (Asteraceae) [81]

- Undet.: Acanthaceae [67]; Asteraceae [109]

40. Aulacorthum (Aulacorthum) spinacaudatum (Kumar \& Burkhardt, 1971)

= Acyrthosiphon (Aulacorthum) spinacaudatum Kumar \& Burkhardt, 1971

- Cucumis sativus L. (Cucurbitaceae) [17, 104]

41. Aulacorthum (Aulacorthum) sp.

- Artemisia sp. (Asteraceae) [19, 20, 70, 82]

- Pterospermum sp. (Malvaceae) [70, 82]

- Solanum sp. (Solanaceae) $[19,70,82]$

42. Aulacorthum (Perillaphis) perillae (Shinji, 1924)

= Perillaphis perillae (Shinji, 1924) [1, 67]

- Artemisia vulgaris L. (Asteraceae) [55]

- Grewia sp. (Tiliaceae) [55]

- Impatiens racemosa DC. (Balsaminaceae) [2]

- Perilla fructescens (L.) Britton (Lamiaceae) [13, 19, 20, 107]

- Perilla sp. (Lamiaceae) [19, 20, 55, 70]

- Prunus sp. (Rosaceae) [17, 37]

- Rhododendron campylocarpum Hook.f. (Ericaceae) [70]

- Undet.: Labiateae [110]; Verbenaceae [70]

43. Brachycaudus (Prunaphis) cardui (Linnaeus, 1758)

= Brachycaudus (Acaudus) cardui (Linnaeus, 1758) : [1, 63, 111]

= Aphis cardui Linnaeus, $1758:$ : [112] 
- Cajanus cajan (L.) Millsp. (Fabaceae) [112]

- Carduus edelbergii Rech.f. (Asteraceae) (= Carduus delbergii) [111]

- Carduus sp. (Asteraceae) [63]

\section{Brachycaudus (Brachycaudus) helichrysi (Kaltenbach, 1843)}

$=$ Anuraphis helichrysi Kaltenbach [50, 113]

= Anuraphis helichrysi var. warei (Theobald, 1923) [114]

= Anuraphis padi (Linnaeus) [115]

= Brachycaudus (Anuraphis) helichrysi (Kaltenbach, 1843) [116]

- Acmella paniculata (Wall. ex DC.) Jansen (=Spilanthes acmella (L.) Murray) (Asteraceae) [78]

- Aesculus indica Colebr. (Hippocastanaceae) [14]

- Ageratum conyzoides L. (Asteraceae) [19, 21, 28, 50, 54, 56, 59, 67, 78, 81, 106]

- Ageratum sp. (Asteraceae) [67, 113, 114]

- Amaranthus sp. (Amaranthaceae) [19]

- Anaphalis araneosa DC. (Asteraceae) [19]

- Anaphalis cinnonomea C.B. Clarke (Asteraceae) [67, 75]

- Anaphalis contorta Hook.f. (Asteraceae) [14, 19]

- Anaphalis marginata L. (Asteraceae) [117]

- Anaphalis nepalensis (DC.) Aswal (Asteraceae) [2]

- Anaphalis sp. (Asteraceae) [67, 75, 78]

- Anaphalis triplinervis Benth. Ex Hance (Asteraceae) [17, 19]

- Artemisia nepalensis Nees (Asteraceae) [106]

- Artemisia sp. (Asteraceae) $[78,118]$

- Artemisia vestita Wall (Asteraceae) [17]

- Artemisia vulgaris L. (Asteraceae) [14, 19]

- Asclepias curassavica L. (Apocynaceae) [81]

- Atropa belladonna L. (Solanaceae) [14, 19]

- Beta vulgaris L. (Chenopodiaceae) [78]

- Bidens bipinnata L. (Asteraceae) [2]

- Bidens pilosa L. (Asteraceae) $[19,78,106]$

- Blumea lacera (Burm.f.) DC. (Asteraceae) [19, 28, 59]

- Blumea mollis (D. Don) Merr. (Asteraceae) [48]

- Blumea sp. (Asteraceae) [50]

- Bomarea caldasii (Kunth) Herb. (Alstroemeriaceae) [28, 59]

- Bougainvillea spectabilis Willd. (Nyctaginaceae) [55]

- Brassica napus L. (Brassicaceae) [19]

- Brassica oleracea L. (Brassicaceae) [19]

- Buddleja sp. (Scrophulariaceae) [19]

- Butea minor Ham. (Fabaceae) [55]

- Cajanus cajan (L.) Millsp. (Fabaceae) [78]

- Calamintha sp. (Lamiaceae) [19]

- Canna sp. (Cannaceae) [106]

- Cerastium fontanum ssp. vulgare (Hartm.) Greuter \& Burdet (=Cerastium vulgatum auct.) (Caryophyliaceae) $[14,19]$

- Cestrum fasciculatum (Schltdl.) Miers (Solanaceae) [19]

- Chenopodium sp. (Chenopodiaceae) [19]

- Chromolaena odorata (L.) King \& H.E. Robins. (Asteraceae) [48]

- Chrysanthemum indicum L. (Asteraceae) [19]

- Chrysanthemum sp. (Asteraceae) [28, 67]

- Cineraria sp. (Asteraceae) [19, 28, 59]

- Cleome gyandra L. (=Cleome pentaphylla L.) (Cleomaceae) [19]

- Clerodendrum sp. (Lamiaceae) [56]

- Cnicus sp. (Asteraceae) [19]

- Cnicus wallichii Hook.f. (Asteraceae) [119] 
- Colocasia sp. (Araceae) [106]

- Conyza angustifolia Horst. Par. ex DC. (Asteraceae) [14]

- Conyza bonariensis (L.) Cronq. (=Erigeron bonariensis L.) (Asteraceae) [120]

- Conyza canadensis (L.) Cronq. (=Erigeron canadensis L.) (Asteraceae) [14, 28]

- Cosmos bipinnatus Cav. (Asteraceae) [14,33]

- Crataeva religiosa Forst.f. (Capparidaceae) [19]

- Crepis sp. (Asteraceae) [78]

- Crotalaria pallida Aiton (=Crotalaria brownie Bertero ex DC.) (Fabaceae) [19]

- Croton sp. (Euphorbiaceae) [31]

- Cryptomeria japonica (L.f.) D.Don (Cupressaceae) [78]

- Cucurbita maxima Duchesne (Cucurbitaceae) [19]

- Cynodon dactylon (L.) Pers. (Poaceae) [78]

- Cynoglossum furcatum Wall. (Boraginaceae) [28]

- Cynoglossum sp. (Boraginaceae) [2]

- Cynoglossum wallichii G. Don (Boraginaceae) [19]

- Cynoglossum wallichii var. glochidiatum (Wall. ex Benth.) Kazmi (=Cynoglossum glochidiatum Wall. ex Benth.) (Boraginaceae) [2]

- Datura metel L. (=Datura fastuosa L.) (Solanaceae) $[19,78]$

- Dichrocephala integrifolia (L.f.) Kuntze (=Dichrocephala latifolia (Pers.) DC.) (Asteraceae) [19]

- Digitalis grandiflora Mill. (Plantaginaceae) [14, 19]

- Digitalis lanata Ehrh. (Plantaginaceae) [14]

- Digitalis purpurea L. (Plantaginaceae) $[14,19]$

- Digitalis sp. (Plantaginaceae) $[19,78]$

- Drymaria cordata (L.) Willd. ex Schult. (Caryophyliaceae) [19, 78]

- Duranta erecta L. (Verbenaceae) [78]

- Dysophylla sp. (Lamiaceae) [19]

- Elephantopus sp. (Asteraceae) [55]

- Eleusine coracana (L.) Gaertn. (Poaceae) [55, 106]

- Engelhardtia sp. (Juglandaceae) [19, 78]

- Erechtites sp. (Asteraceae) [78, 106]

- Erechtites valerianaefolia DC. (Asteraceae) [19]

- Erigeron sp. (Asteraceae) [19]

- Eupatorium adenophorum Spreng. (Asteraceae) [50, 78]

- Eupatorium odoratum L. (Asteraceae) [50, 55, 78, 81, 106]

- Eupatorium sp. (Asteraceae) [21]

- Eupatorium wallichii DC. (Asteraceae) [19]

- Euphorbia hirta L. (Euphorbiaceae) [19]

- Galinsoga parviflora Cav. (Asteraceae) [78]

- Gamochaeta purpurea (L.) Cabrera (=Gnaphalium purpurium L.) (Asteraceae) [19]

- Gardenia jasminoides J. Ellis (=Gardenia florida L.) (Rubiaceae) [19]

- Geranium ocellatum Cambess. (Geraniaceae) [19]

- Gerbera sp. (Asteraceae) [28, 121]

- Glebionis coronaria (L.) Cass. ex Spach (=Chrysanthemum coronarium L.) (Asteraceae) [78]

- Gnaphalium sp. (Asteraceae) [75]

- Gonostegia hirta (Hassk.) Miq. (=Pouzolzia hirta Blume ex Hassk.) (Urticaceae) [78]

- Gynura crepidioides Benth. (Asteraceae) [50, 122]

- Gynura cusimbua (D. Don) S. Moore (=Gynura angulosa DC.) (Asteraceae) [58, 54]

- Gynura nepalensis DC. (Asteraceae) $[19,81]$

- Helianthus annuus L. (Asteraceae) [55, 19]

- Heliotropium indicum L. (Boraginaceae) $[14,19]$

- Hibiscus sp. (Malvaceae) [19]

- Holmskioldia sanguinea Retz. (Verbenaceae) [19]

- Hypochaeris glabara L. (Asteraceae) [81]

- Inula cappa (Buch.-Ham. Ex D. Don) DC. (Asteraceae) [14] 
- Inula sp. (Asteraceae) [2]

- Jatropha curcas L. (Euphorbiaceae) [55]

- Jatropha sp. (Euphorbiaceae) [17]

- Lablab purpureus (L.) Sweet (=Dolichos lablab L.) (Fabaceae) [123]

- Lactuca sp. (Asteraceae) [19]

- Laggera flava (DC.) Benth. (Asteraceae) [55]

- Laggera pterodonta (DC.) Benth. (Asteraceae) [14]

- Laggera sp. (Asteraceae) [55]

- Lantana camara L. (Verbenaceae) [19]

- Leucaena leucocephala (Lam.) deWit.) (=Leucaena glauca auct.) (Fabaceae) [78]

- Levisticum officinale W.D.J. Koch (Apiaceae) [14]

- Lycopersicon esculentum Mill. (Solanaceae) [17, 19, 70, 78]

- Maesa chisia Buch.-Ham. ex D. Don (Maesaceae) [78]

- Maesa sp. (Maesaceae) [19]

- Malus domestica Borkh. (=Pyrus malus L.) (Rosaceae) [2]

- Mangifera indica L. (Anacardiaceae) [55]

- Mikania scandens (L.) Willd. (Asteraceae) [19]

- Morus alba L. (=Morus indica L.) (Moraceae) [55]

- Musa sp. (Musaceae) [106]

- Nicotiana tabacum L. (Solanaceae) $[14,19]$

- Notonia grandiflora DC. (Asteraceae) [51]

- Osbeckia chinensis L. (Melastomaceae) [78, 55]

- Oxalis corniculata L. (Oxalidaceae) [14, 19]

- Papaver orientale L. (Papaveraceae) [14]

- Passiflora foetida L. (Passifloraceae) [19]

- Pedicularis gracilis Wall. ex Benth. (Orobanchaceae) [17]

- Persicaria barbata (L.) H. Hara (=Polygonum barbatum L.) (Polygonaceae) [78]

- Persicaria chinensis (L.) H. Gross (=Polygonum chinense L.) (Polygonaceae) [19]

- Petunia integifolia (Hook) Schinz \& Thell. (=Petunia violacea Lindl.) (Solanaceae) [14]

- Petunia sp. (Solanaceae) [19]

- Philadelphus coronaries L. (Hydrangeaceae) [19]

- Photinia sp. (Rosaceae) [19]

- Pogostemon sp. (Lamiaceae) [55]

- Polygonum sp. (Polygonaceae) [14, 19]

- Prunus armeniaca L. (Rosaceae) $[28,39,55]$

- Prunus cerasoides Buch.-Ham. ex D.Don (=Prunus puddum (Rox. Ex Ser.)) (Rosaceae) [55]

- Prunus cerasus L. (Rosaceae) [19]

- Prunus cornuta (Wall. ex Royle) Steud. (Rosaceae) [124]

- Prunus domestica L. (Rosaceae) [14, 19, 28, 39, 55, 59]

- Prunus dulcis (Mill.) D.A. Webb. (=Prunus amygdalus Batsc) (Rosaceae) [28, 39, 75]

- Prunus padus L. (Rosaceae) [28]

- Prunus persica (L.) Batsch (Rosaceae) $[14,17,19,28,39,50,55,59,115,106,116]$

- Prunus persica (L.) var.flordasun (Rosaceae) [71]

- Prunus salicina Lindl. (=Prunus triflora Roxb.) (Rosaceae) [19]

- Prunus sp. (Rosaceae) [22, 67, 70, 78]

- Pseudognaphalium luteoalbum (L.) Hilliard \& Burtt (=Gnaphalium luteoalbum L.; Gnaphalium album auct.nonn.; Anaphalis luteoalbum misident.) (Asteraceae) [14, 19, 124]

- Punica granatum L. (Punicaceae) [17, 19]

- Pyrus sp. (Rosaceae) [75]

- Quercus sp. (Fagaceae) [19]

- Rhododendron arboreum Sm. (Ericaceae) [19]

- Rhododendron sp. (Ericaceae) [14]

- Rosa webbiana Wall. ex Royle (Rosaceae) [125]

- Rosa sp. (Rosaceae) [14, 78] 
- Rubia cordifolia L. (Rubiaceae) [14, 19]

- Rubus ellipticus Sm. (Rosaceae) [14, 19]

- Rubus sp. (Rosaceae) [19]

- Rudbeckia tagetes James (=Rudbeckia tageteiodes (auct.) (Asteraceae) [106]

- Rumex nepalensis Spreng. (Polygonaceae) [78]

- Salix sp. (Salicaceae) [55]

- Salvia sp. (Lamiaceae) [19]

- Sambucus javanica Reinw. ex Blume (Adoxaceae) [19]

- Saussurea costus (Falc.) Lipsch. (=Saussurea lappa (Dacne.) C.B. Clarke) (Asteraceae) [17, 39, 41]

- Schima wallichii (DC.) Korth. (Theaceae) [19]

- Senebiera pinnatifida DC. (Brassicaceae) [19]

- Senecio alatus Wall. ex DC. (Asteraceae) [14]

- Senecio chrysanthemoides DC. (Asteraceae) $[2,17]$

- Senecio densiflorus Wall. ex DC. (Asteraceae) [14]

- Senecio diversiflolius (Hook. \& Arn.) DC. (Asteraceae) [14]

- Senecio scandens Buch.-Ham. ex D. Don (Asteraceae) [19, 78]

- Senecio setulosus Phil. (Asteraceae) [14]

- Senecio sp. (Asteraceae) [55]

- Solanum erianthum D. Don (=Solanum verbascifolium L.) (Solanaceae) [19]

- Solanum melongena L. (Solanaceae) [55]

- Solanum multifidum Lam. (=Solanum pinnatifidum Ruiz. \& Pav.) (Solanaceae) [19]

- Solanum sp. (Solanaceae) [19]

- Solanum tuberosum L. (Solanaceae) $[14,55]$

- Solanum virginianum L. (=Solanum xanthocarpum Schrad.) (=Solanum surattense Burm. f.) (Solanaceae) [14]

- Solanum xanti A. Gray (Solanaceae) [14]

- Solidago sp. (Asteraceae) [2]

- Solidago virgaurea L. (Asteraceae) [14]

- Sonchus arvensis L. (Asteraceae) [78]

- Sonchus sp. (Asteraceae) [19]

- Sorbus vestita (Wall. ex G. Don) Lodd. (=Pyrus vestita Wall ex G. Don) (Rosaceae) [2]

- Spiraea bella Sims. (Rosaceae) [2]

- Spiraea corymbosa Raf. (Rosaceae) [19]

- Symplocos cratigeoides Buch.-Ham. ex D. Don (Symplocaceae) [19]

- Tephrosia sp. (Fabaceae) [2]

- Tridax procumbens L. (Asteraceae) [19, 78]

- Triumfetta pilosa Roth (Tiliaceae) [78]

- Urtica parviflora Roxb. (Tiliaceae) [126]

- Vigna unguiculata (L.) Walp. ssp. cylindrica (L.) Verdc. (=Vigna catjang (Burm.f.) Walp.) (Fabaceae) [19]

- Vinca sp. (Apocynaceae) [17, 19]

- Vitex sp. (Lamiaceae) [19]

- Xerochrysum bracteatum (Vent.) Tzvelev (=Helichrysum bracteatum (Vent.) Andrews) (Asteraceae) [81]

- Undet.: Acanthaceae [127]; Araceae [78]; Asteraceae [14, 38]; Urticaceae [14]; indet. Families $[17,95]$

45. Brachycaudus (Brachycaudus) sp.

- Anaphalis cinnonomea C.B. Clarke (Asteraceae) [75]

- Anaphalis sp. (Asteraceae) [75]

- Gnaphalium sp. (Asteraceae) [75]

46. Brachycaudus (Mordvilkomemor) pilosus (Mordvilko ex Nevsky, 1929)

- Undet.: Asteraceae [40] 
47. Brachycaudus (Mordvilkomemor) sp.

= Mordvilkomemor sp. [39, 128, 129]

- Artemisia vestita Wall (Asteraceae) [39, 128]

48. Brachycaudus (Scrophulaphis) persicae (Passerini, 1860)

=Brachycaudus (Acaudus)persicae (Passerini) [71]

- Prunus dulcis (Mill.) D.A.Webb (= Prunus dulcis (Miller)) [71]

- Prunus persica (L.) Batsch (Rosaceae) [71]

49. Brachycaudus (Thuleaphis) amygdalinus (Schouteden, 1905)

= Thuleaphis amygdalinus Schouteden, 1905 [14]

= Thuleaphis ?amygdalinus Schouteden, 1905 [105]

- Fagopyrum acutatum (Lehm.) Mansf. ex K.Hammer (=Fagopyrum cymosum (Trev.) Meisn) (Polygonaceae) [14, 105]

- Nicotiana sp. (Solanaceae) [14]

50. Brachycaudus (Thuleaphis) rumexicolens (Patch, 1917)

= Thuleaphis rumexicolens Patch, 1917 [39, 64, 130]

- Fagopyrum acutatum (Lehm.) Mansf. ex K.Hammer (=Fagopyrum cymosum (Trev.) Meisn) (Polygonaceae) [64]

- Rumex hastatulus Baldwin (Polygonaceae) [17, 23, 39]

- Rumex nepalensis Spreng. (Polygonaceae) [17]

\section{Brachycaudus (Thuleaphis) sp.}

- Rumex hastatulus Baldwin (Polygonaceae) [131]

52. Brachymyzus jasmini Basu, 1964

- Eleusine sp. (Poaceae) [19]

- Jasminum humile L. (Oleaceae) [19, 20]

- Nellia sp. (Rosaceae) [70, 78]

- Pilea macrocarpa (L.) Liebm. (Urticaceae) [70, 78]

53. Brachysiphoniella montana (van der Goot, 1917)

= Brachycolus gramini Takahashi, 1920 [114]

= Semiaphis (Brachysiphoniella) montana (van der Goot, 1917) [132]

- Cynodon dactylon (L.) Pers. (Poaceae) [133]

- Cynodon plectostachyus (K. Schum.) Pilg. (Poaceae) [28, 121]

- Cynodon sp. (Poaceae) [50]

- Eleusine sp. (Poaceae) [107]

- Leersia sp. (Poaceae) [48]

- Miscanthus sp. (Poaceae) [48]

- Pennisetum sp. (Poaceae) [48]

- Phragmites sp. (Poaceae) [48]

- Poa sp. (Poaceae) [107]

54. Brevicoryne barbareae Nevsky, 1929

- Barbarea vulgaris R. Br. (Brassicaceae) [17, 39, 41, 134]

- Brassica sp. (Brassicaceae) [134]

- Nasturtium sp. (Brassicaceae) [17, 39, 41, 134]

- Raphanus sativus L. (Brassicaceae) [134]

55. Brevicoryne brassicae (Linnaeus, 1758)

=Aphis brassicae Linnaeus, 1758 [112]

- Atropa belladonna L. (Solanaceae) [14]

- Beta vulgaris L. (Chenopodiaceae) [19, 20, 134]

- Brassica juncea L. Czern. (Brassicaceae) 14, 28, 134]

International J. Research Studies in Zoology (IJRSZ) 
- Brassica napus L. (Brassicaceae) [14, 19, 20, 55, 134]

- Brassica nigra (L.) W.D.J. Koch (Brassicaceae) 52, 57]

- Brassica oleracea L. (Brassicaceae) [14, 17, 19, 20, 30, 39, 55, 60, 70, 134]

- Brassica oleracea L. var. botrytis L. (=Brassica oleracea var. cauliflora (misnomen.) (Brassicaceae) $[28,39,45,50]$

- Brassica oleracea L. var. capitata L. (Brassicaceae) [28, 39, 45, 50, 60]

- Brassica oleracea L. var. gongyloides L. (Brassicaceae) [50]

- Brassica oleracea L. var. viridis (=Brassica oleracea var. acephala) DC.) (Brassicaceae) [72]

- Brassica oleracea L. var. kashmiriana Naqshi \& Javeid (Brassicaceae) [71]

- Brassica rapa L. (Brassicaceae) [28]

- Brassica rapa ssp. dichotoma (Roxb.) Hanelt (=Brassica dichotoma misnomen.) (Brassicaceae) [36]

- Brassica rapa ssp. campestris (L.) A.R. Clapham (= Brassica campestris L.) (Brassicaceae) [14, $19,20,28,32,50,60,134]$

- Brassica sp. (Brassicaceae) [78, 14, 33, 45, 70, 106, 131]

- Cajanus cajan (L.) Millsp. (Fabaceae) [112]

- Capsella bursapastoris (L.) Medik) (Brassicaceae) [20]

- Cardamine hirsuta L. (Brassicaceae) [20, 134]

- Cardamine impatiens L. (Brassicaceae) [14]

- Cleome gyandra L. (=Cleome pentaphylla L.; Gynandropsis pentaphylla (L.) DC) (Cleomaceae) [48]

- Ficus sp. (Moraceae) [14]

- Iberis sp. (Brassicaceae) $[135,136]$

- Lycopersicon esculentum Mill. (Solanaceae) [56]

- Matthiola incana (L.) W. T. Aiton (=Guettarda incana misident.) (Brassicaceae) [14]

- Nasturtium officinale R. Br. (Brassicaceae) [2]

- Petunia integifolia (Hook) Schinz \& Thell. (=Petunia violacea Lindl.) (Solanaceae) [14]

- Raphanus sativus L. (Brassicaceae) [14, 19, 20, 28, 33, 45, 112, 131, 134]

- Scutellaria scandens Buch.-Ham. ex D. Don (Lamiaceae) [14]

- Solanum tuberosum L. (Solanaceae) [14, 39]

- Solanum virginianum L. (=Solanum xanthocarpum Schrad.; Solanum surattense Burm. f.) (Solanaceae) [14]

- Veronica agrestis L. (Plantaginaceae) [17]

- Zea mays L. (Poaceae) [17]

- Undet.: Brassicaceae [14, 39]; Lamiaceae, Moraceae, Solanaceae [134]

56. Brevicoryne capsici Sathe \& Jadhav, 2008§

- Capsicum annuum Linn. (Solanaceae) [77]

57. Capitophorus archangelskii Nevsky, 1928

- Tricholepis furcata DC. (Asteraceae) [15]

58. Capitophorus carduinus (Walker, 1850)

- Anemone rivularis Buch.-Ham. ex DC. (Ranunculaceae) [19, 20, 136]

- Cnicus sp. (Asteraceae) [19, 20, 34, 136]

- Cnicus wallichii Hook.f. (Asteraceae) [123, 137]

- Cosmos sp. (Asteraceae) [19]

- Geranium sp. (Geraniaceae) $[19,101]$

- Osbeckia capitata Bentm. Ex Naudin (Melastomaceae) [19]

- Rubus ellipticus Sm. (Rosaceae) [19]

59. Capitophorus cirsiiphagus Takahashi, 1961

- Cnicus sp. (Asteraceae) [132] 
60. Capitophorus elaeagni (del Guercio, 1894)

- Carduus edelbergii Rech.f. (Asteraceae) [87]

- Cnicus involucratus Wall. ex DC. (Asteraceae) [50]

- Cnicus sp. (Asteraceae) [19, 20, 78, 136]

- Cnicus wallichii Hook.f. (Asteraceae) [137]

- Cousinia thomsoni Clarke (=Cnicus thomsoni auct. nonn.) (Asteraceae) $[2,137]$

- Cynara cardunculus L. (=Cynara scolymus L.) (Asteraceae) [28, 138]

- Polygonum viscosum Buch.-Ham. ex D. Don (Polygonaceae) [19]

- Swertia sp. (Gentianaceae) [88]

61. Capitophorus formosartemisiae (Takahashi, 1921)

- Anaphalis cinnonomea C.B. Clarke (Asteraceae) [2]

- Artemisia dentata Willd. (Asteraceae) $[19,35,36]$

- Artemisia nepalensis Nees (Asteraceae) [106, 139]

- Artemisia roxburghiana Bess (Asteraceae) [21]

- Artemisia sp. (Asteraceae) [17, 19, 20, 70, 78, 136]

- Artemisia vulgaris L. (Asteraceae) [14, 16, 19, 21, 50, 55, 78, 105, 106, 139]

- Chrysanthemum indicum L. (Asteraceae) [106]

- Cosmos sp. (Asteraceae) [2]

- Erigeron sp. (Asteraceae) [19]

- Inula orientalis Lam. (=Inula grandiflora Willd.) (Asteraceae) [2]

- Inula sp. (Asteraceae) [2]

- Maesa indica (Roxb.) A. DC. (Maesaceae) [34, 136]

- Musa paradisiaca L. (Musaceae) [2]

- Polygonum sp. (Polygonaceae) [19]

- Saussurea albescens Hook.f. \& Thoms. ex C.B. Clarke (Asteraceae) [2]

- Saussurea costus (Falc.) Lipsch. (=Saussurea lappa (Dacne.) C.B. Clarke) (Asteraceae) [34]

- Senecio triligulatus Ham. (Asteraceae) [55]

62. Capitophorus himachali Chakrabarti \& Maity, 1980

- Polygonum sp. (Polygonaceae) [140]

63. Capitophorus himalayensis Ghosh, Ghosh \& Raychaudhuri, 1971

- Hippophae sp. (Elaeagnaceae) [2, 137]

- Unidentified plants (Polygonaceae) [20, 136]

64. Capitophorus hippophaes hippophaes (Walker, 1852)

- Persicaria barbata (L.) H. Hara (=Polygonum barbatum L.) (Polygonaceae) [25]

- Hippophae sp. (Elaeagnaceae) [141]

- Persicaria chinensis (L.) H. Gross (=Polygonum chinense L.) (Polygonaceae) [36, 122, 125]

- Persicaria hydropiper (L.) Delarbre (=Polygonum hydropiper L.) (Polygonaceae) [96]

- Polygonum sp. (Polygonaceae) [50]

- Spermadictyon sauveolens Roxb. (=Hamiltonia sauveolens auct. nonn.) (Rubiaceae) [19]

65. Capitophorus hippophaes javanicus Hille Ris Lambers, 1953

- Aconogonon molle (D. Don) H. Hara (=Polygonum molle D. Don.) (Polygonaceae) [78]

- Ageratum conyzoides L. (Asteraceae) [19]

- Eupatorium wallichii DC. (Asteraceae) [19]

- Mirabilis jalapa L. (Nyctaginaceae) [19]

- Persicaria barbata (L.) H. Hara (=Polygonum barbatum L.) (Polygonaceae) [14, 19, 20, 64, 78, 81, 136]

- Persicaria chinensis (L.) H. Gross (=Polygonum chinense L.) (Polygonaceae) [55]

- Persicaria glabra (Willd.) M. Gónez (=Polygonum glabrum Willd.) (Polygonaceae) [19, 20, 136]

- Persicaria hydropiper (L.) Delarbre (=Polygonum hydropiper L.) (Polygonaceae) [19, 20, 34, 55, 136] 
- Persicaria mollis (D. Don). Don) H. Gross (=Polygonum paniculatum Blume) (Polygonaceae) [13]

- Persicaria orientale (L.) Spatch (=Polygonum orientale L.) (Polygonaceae) $[19,58,78]$

- Persicaria perfoliata (L.) H. Gross (=Polygonum perfoliatum L.) (Polygonaceae) $[19,136]$

- Persicaria pubescens (Blume) H. Hara (=Polygonum flaccidum auct.) (Polygonaceae) [16]

- Polygonum alatum Buch.-Ham. ex D. Don (Polygonaceae) [19, 20, 78, 136]

- Polygonum flaccidum Roxb. (=Polygonum serrulatum Lagasca) (Polygonaceae) [19, 20, 34, 136]

- Polygonum sp. (Polygonaceae) [14, 17, 20, 35, 36, 50, 67]

66. Capitophorus litanensis Raychaudhuri, Singh \& Raychaudhuri, 1984

- Bidens sp. (Asteraceae) [142]

67. Capitophorus meghalayensis Basu \& Raychaudhuri, 1976

$=$ Capitophorus archangelskii Nevsky, 1928* [22]

- Elaeagnus sp. (Elaeagnaceae) [20, 22, 70, 136]

68. Capitophorus mitegoni Eastop, 1956

= Capitophorus indicus Ghosh \& Raychaudhuri, 1968 [1]

= Capitophorus hippophaes indica Ghosh \& Raychaudhuri, 1968 [79, 109]

= Capitophorus hippophaes mitegoni Eastop, 1956: [1, 108]

= Capitophorus polygoni Ghosh, Ghosh \& Raychaudhuri, 1971 [1]

- Aconogonon molle (D. Don) H. Hara (=Polygonum molle D. Don.) (Polygonaceae) [19, 20, 78, 136]

- Amaranthus cruentus L. (= Amaranthus paniculatus L.) (Amaranthaceae) [2]

- Boehmeria sp. (Urticaceae) [19]

- Cosmos sp. (Asteraceae) [31]

- Desmodium sp. (Fabaceae) [19]

- Eupatorium wallichii DC. (Asteraceae) [19]

- Ipomoea sp. (Convolvulaceae) [19]

- Lamium album L. (Lamiaceae) [2]

- Persicaria barbata (L.) H. Hara (=Polygonum barbatum L.) (Polygonaceae) [78]

- Persicaria chinensis (L.) H. Gross (=Polygonum chinense L.) (Polygonaceae) [54, 55, 136, 143]

- Persicaria glabra (Willd.) M. Gónez (=Polygonum glabrum Willd.) (Polygonaceae) [19]

- Persicaria hydropiper (L.) Delarbre (=Polygonum hydropiper L.) (Polygonaceae) [144, 145]

- Persicaria mollis (D. Don). Don) H. Gross (=Polygonum paniculatum Blume) (Polygonaceae) [13]

- Persicaria nepalensis (Meisn.) H. Gross (=Polygonum nepalense Meisn.) (Polygonaceae) [20]

- Persicaria orientale (L.) Spatch (=Polygonum orientale L.) (Polygonaceae) $[19,20,136]$

- Persicaria perfoliata (L.) H. Gross (=Polygonum perfoliatum L.) (Polygonaceae) [20, 136]

- Polygonum alatum Buch.-Ham. ex D. Don (Polygonaceae) [19, 20, 136]

- Polygonum sinensis P.E. Resreratrol (Polygonaceae) [20]

- Polygonum sp. (Polygonaceae) [17, 32, 55, 78, 106, 108]

- Rumex nepalensis Spreng. (Polygonaceae) [19, 20, 136]

- Rumex sp. (Polygonaceae) [19]

- Undet.: Polygonaceae) [20, 136]

69. Capitophorus similis van der Goot, 1915

- Aralia thomsoni L. (Araliaceae) [19]

70. Capitophorus tricholepidis Chakrabarti, 1976

- Tricholepis furcata DC. (Asteraceae) [146]

71. Capitophorus sp.

- Anaphalis cinnonomea C.B. Clarke (Asteraceae) [75]

- Anaphalis sp. (Asteraceae) [75]

- Artemisia sp. (Asteraceae) $[63,75]$

- Artemisia vestita Wall (Asteraceae) [75]

- Artemisia vulgaris L. (Asteraceae) [50,75]

- Brassica oleracea var. botrytis L. (Brassicaceae) [50]

- Centaurea iberica Trevir. ex Spreng. (Asteraceae)[125] 
- Gnaphalium sp. (Asteraceae) [75]

- Hippophae salicifolia D. Don (Elaeagnaceae) [17, 39]

- Leucosceptrum cannum Sm. (Lamiaceae) [50]

- Osbeckia crinata Benth. (Melastomaceae) [50]

- Polygonum sp. (Polygonaceae) [50]

72. Carolinaia (Juncomyzus) scirpi (van der Goot, 1917)

= Aulacorthum (Aulacorthum) scirpi van der Goot, 1917: [1]

- Scleria cochinchinensis (Lour.) Druce (=Scleria elata Thwaites) (Cyperaceae) [20, 147]

73. Carolinaia (Juncomyzus) sp.

- Scleria cochinchinensis (Lour.) Druce (=Scleria elata Thwaites) (Cyperaceae) [19]

74. Cavariella (Cavaraiellia) aquatica (Gillette \& Bragg, 1916)

- Hordeum vulgare L. (Poaceae) [71]

- Salix elaeagnos Scop. (=Salix elegans auct. non.) (Salicaceae) [76]

- Salix sp. (Salicaceae) [17, 39, 41, 70]

75. Cavariella (Cavariella) ? konoi Takahashi, 1939

- Geranium lucidum L. (Geraniaceae) [17]

- Pimpinella diversifolia DC. (Apiaceae) [17]

- Pimpinella umbellata ? (Apiaceae) [17]

76. Cavariella (Cavariella) aegopodii (Scopoli, 1763)

- Geranium sp. (Geraniaceae) [37]

- Glochidion sp. (Phyllanthaceae) [132]

- Heracleum sosnowskyi Manden (Apiaceae) [19, 20]

- Heracleum sp. (Apiaceae) [14, 105]

- Pimpinella diversifolia DC. (Apiaceae) [14, 17, 19, 20]

- Polygonum sp. (Polygonaceae) [67]

- Salix aegyptiaca L. (Salicaceae) [98]

- Salix babylonica L. (Salicaceae) [19, 20, 132]

- Salix cinerea L. (=Salix acuminata Mill.) (Salicaceae) [14]

- Salix denticulata Andersson (Salicaceae) [148]

- Salix hastata L. (Salicaceae)[75]

- Salix sp. (Salicaceae) $[67,96,98]$

- Salix tetrasperma Roxb. (Salicaceae) [2, 149]

- Sanicula europaea L. (Apiaceae) [14, 19, 20]

- Zingiber officinale Rosc. (Zingiberaceae) [34]

- Undet.: Apiaceae) [14, 67]

77. Cavariella (Cavariella) araliae Takahashi, 1921

- Aralia sp. (Araliaceae) [150]

- Aralia thomsoni L. (Araliaceae) $[19,20]$

- Salix sp. (Salicaceae) [19, 20, 78]

78. Cavariella (Cavariella) biswasi Ghosh, Basu \& Raychaudhuri, 1969

- Heracleum canadicans Wall. ex DC. (Apiaceae) [87]

- Salix babylonica L. (Salicaceae) $[19,20]$

- Salix elaeagnos Scop. (=Salix elegans auct. nonn.) (Salicaceae) $[19,20]$

- Salix sp. (Salicaceae) [19, 20]

79. Cavariella (Cavariella) himachali Ghosh, 1986

- Indigofera dregeana F. Mey (=Indigofera gerardiana Wall. ex Baker) (Fabaceae) [37]

- Spiraea canescens D. Don (Rosaceae) [37]

80. Cavariella (Cavariella) indica Maity \& Chakrabarti, 1982

- Populus ciliata Wall. ex Royle (Salicaceae) [2]

- Salix babylonica L. (Salicaceae) [76, 85]

- Salix sp. (Salicaceae) [76]

International J. Research Studies in Zoology (IJRSZ) 
81. Cavariella (Cavariella) konoi Takahashi, 1939

- Salix babylonica L. (Salicaceae) [2]

82. Cavariella (Cavariella) nigra Basu, 1964

- Salix denticulata Andersson (Salicaceae) [148]

- Salix elaeagnos Scop. (=Salix elegans auct. nonn.) (Salicaceae) [19, 20]

- Undet.: Apiaceae) $[17,110]$

83. Cavariella (Cavariella) salicicola (Matsumura, 1917)

- Gnaphalium sp. (Asteraceae) [20,55, 150]

- Salix babylonica L. (Salicaceae) [19, 20, 132]

- Salix drupifolia ? (Salicaceae) $[55,148]$

- Salix sp. (Salicaceae) [20, 55, 106, 151]

- Sanicula europaea L. (Apiaceae) [17, 130]

84. Cavariella (Cavariella) salicis (Monell, 1879)

- Populus ciliata Wall. ex Royle (Salicaceae) [2]

- Salix denticulata Andersson (Salicaceae) [2]

- Salix elaeagnos Scop. (=Salix elegans auct. nonn.) (Salicaceae) [76]

- Salix sp. (Salicaceae) [2]

85. Cavariella (Cavariella) simlaensis Chowdhuri, Basu \& Raychaudhuri, 1969

- Sanicula europaea L. (Apiaceae) [17, 130]

86. Cavariella Cavariella) sp.

- Salix eriophylla Andersson (Salicaceae) [152]

- Salix sp. (Salicaceae) [31]

- Undet.: Solanaceae) $[19,20,70]$

87. Ceruraphis eastopi Hille Ris Lambers, 1966

- Viburnum cotinifolium D. Don (Adoxaceae) [2, 153]

- Viburnum sp. (Adoxaceae) [154]

88. Chaetomyzus rhododendri Ghosh \& Raychaudhuri, 1962

- Rhododendron arboreum Sm. (Ericaceae) [2, 155]

- Rhododendron sp. (Ericaceae) [90]

89. Chaetosiphon (Chaetosiphon) chaetosiphon (Nevsky, 1928)

- Rosa sp. (Rosaceae) [156]

90. Chaetosiphon (Chaetosiphon) gracilicorne David, Rajasingh \& Narayanan, 1970 (1971)

= Chaetosiphon chaetosiphon indicum Chakrabarti \& Ghosh, 1970 [14, 64, 157, 158]

- Artemisia vulgaris L. (Asteraceae) [14]

- Rosa macrophylla Lindl. (Rosaceae) $[17,156]$

- Rosa moschata Herrm. (Rosaceae) [14, 17]

- Rosa webbiana Wall. ex Royle (Rosaceae) [125]

- Rosa sp. (Rosaceae) [14, 17, 39, 64, 67]

91. Chaetosiphon (Chaitomyzus) heterotricum Chakrabarti, Ghosh \& Raychaudhuri, 1971

= Chaetosiphon (Pentatrichopus) heterotrichum Chakrabarti, Ghosh \& Raychaudhuri, 1971 [14, 64]

- Lamium sp. (Lamiaceae) [137]

- Salvia glutinosa L. (Lamiaceae) $[2,73]$

- Salvia sp. (Lamiaceae) [14, 42, 64]

92. Chaetosiphon (Pentatrichopus) fragaefolii (Cockerell, 1901)

- Fragaria sp. (Rosaceae) [17, 156] 
93. Chaetosiphon (Pentatrichopus) glabrum David, Rajasingh \& Narayanan, 1970 (1971)

- Rosa brunonii Lindl. (Rosaceae) [87]

- Rosa macrophylla Lindl. (Rosaceae) [17, 156]

- Rosa sp. (Rosaceae) [17]

- Rubus fruticosus Sm. (Rosaceae) [71]

- Rubus plicatus Weihe \& Nees (=Rubus fruticosus L.) (Rosaceae) [87]

94. Chaetosiphon (Pentatrichopus) tetrarhodum (Walker, 1849)

- Rosa brunonii Lindl. (Rosaceae) [2]

- Rosa macrophylla Lindl. (Rosaceae) [72]

- Rosa moschata Herrm. (Rosaceae) [17]

- Rosa sp. (Rosaceae) [14, 17, 51, 64, 121]

95. Chaetosiphon (Pentatrichopus) sp.

- Rosa sp. (Rosaceae) [28]

96. Chakrabartiaphis hydrangeae (Chakrabarti \& Bhattacharya, 1982)

= Tubicauda hydrangeae Chakrabarti \& Bhattacharya 1982

- Hydrangea sp. (Hydrangeaceae) [159]

97. Coloradoa artemisiae (del Guercio, 1913)

- Artemisia vulgaris L. (Asteraceae) [56, 132, 150]

- Justicia adhatoda L. (=Adhatoda vasica Nees) (Acanthaceae) [55]

98. Coloradoa indica Verma, 1974

- Artemisia sp. (Asteraceae) [88, 160]

99. Coloradoa kashmirica Rishi \& Lone, 1985

- Artemisia sylvatica Maxim. (Asteraceae) [161]

100. Coloradoa rufomaculata (Wilson, 1908)

- Artemisia sp. (Asteraceae) [19, 20]

- Artemisia vulgaris L. (Asteraceae) [19, 20, 34]

- Chrysanthemum indicum L. (Asteraceae) [70, 162]

- Chrysanthemum morifolium Ramat (=Chrysanthemum sinense Sabine ex Sweet) (Asteraceae) [28]

- Chrysanthemum sp. (Asteraceae) [51, 59, 67, 131]

- Glebionis coronaria (L.) Cass. ex Spach (=Chrysanthemum coronarium L.) (Asteraceae) [19, 20, 34]

- Polygonum sp. (Polygonaceae) [67]

101. Cryptaphis garhwalensis Bhattacharya, Mondal \& Chakrabarti, 1983

- Lamium album L. (Lamiaceae) [163]

102. Cryptaphis rostrata Chakrabarti \& Raychaudhuri, 1974

- Undet.: Lamiaceae) [110]

103. Cryptaphis salviae Bhattacharya \& Dey, 1996

- Salvia leucantha Cavanilles (Lamiaceae) [164]

104. Cryptomyzus (Cryptomyzus) taoi Hille Ris Lambers, 1963

= Cryptomyzus taoi indicus Ghosh \& Raychaudhuri, 1972 [147]

- Artemisia sp. (Asteraceae) [106, 165]

- Craniotome versicolor Reichenbach (Lamiaceae) [2]

- Leonurus sibiricus L. (Lamiaceae) [19, 20, 147]

- Leucas aspera (Willd.) Link (Lamiaceae) [19, 20, 55, 147]

- Youngia japonica (L.) DC. (=Crepis japonica (L.) Benth.) (Asteraceae) [19]

International J. Research Studies in Zoology (IJRSZ) 
105. Cryptosiphum aertemisiae Buckton, 1879

- Artemisia sp. (Asteraceae) [19, 20, 78, 152]

- Artemisia vestita Wall (Asteraceae) [166]

- Artemisia vulgaris L. (Asteraceae) [19, 55, 78, 106, 107, 165]

- Sonchus sp. (Asteraceae) [78]

106. Dysaphis (Cotoneasteria) microsiphon (Nevsky, 1929)

= Dysaphis (Dysaphis) montemartinii (del Guercio, 1913)

= Dysaphis (Dysaphis) montemartinii ghanii Stroyan, 1963 [70]

= Dysaphis ghanii Stroyan, 1963 [17, 30, 33]

= Dysaphis (Dysaphis) stroyani Ghosh, 1986 [37]

- Artemisia sp. (Asteraceae) [167]

- Artemisia vestita Wall (Asteraceae) [166]

- Artemisia vulgaris L. (Asteraceae) [2,167]

- Brassica oleracea L. (Brassicaceae) [30, 33, 167]

- Chenopodium album L. (Chenopodiaceae) [2]

- Cotoneaster bacillaris Wall. ex Lindl. (Rosaceae) [17, 30, 70]

- Euonymus hamiltonianus Wall. (Celastraceae) [167]

- Fragaria nilgerrensis Schltdl. ex J. Gay (Rosaceae) [70]

- Fragaria sp. (Rosaceae) [17, 30, 167]

- Geranium sp. (Geraniaceae) [167]

- Girardinia diversifolia (Link) Friis (=Girardinia heterophylla (Decne.)) (Urticaceae) [167]

- Gnaphalium sp. (Asteraceae) [167]

- Lactuca dolichophylla Kitam. (=Lactuca longifolia DC.) (Asteraceae) [167]

- Lyonia ovalifolia (Wall.) Drude (=Pieris ovalifolis (Wall. D. Don)) (Ericaceae) [16]

- Mazus surculosus D. Don (Phrymaceae) [167]

- Pedicularis gracilis Wall. ex Benth. (Orobanchaceae) [37, 167]

- Pennisetum glaucum (L.) R. Br. (=Setaria glauca (L.) P. Beauv.) (Poaceae) $[14,16]$

- Persicaria wallichii Grentor \& Burdet (=Polygonum polystachyum Wall ex Meisn.) (Polygonaceae) [167]

- Populus ciliata Wall. ex Royle (Salicaceae) [37]

- Rumex nepalensis Spreng. (Polygonaceae) [167]

- Salix sp. (Salicaceae) [37]

- Taraxacum officinale aggr. (Dandelion) (Asteraceae) [167]

- Viburnum sp. (Adoxaceae) [17, 37]

107. Dysaphis (Dysaphis) atina Ghosh, Basu \& Raychaudhuri, 1969

- Anaphalis busua (Buch.-Ham.) DC. (Asteraceae) [2]

- Carduus onopordioides Fisch. ex Bieb. (Asteraceae) [87]

- Cnicus wallichii Hook.f. (Asteraceae) $[15,17]$

108. Dysaphis (Dysaphis) branchoi (Börner, 1950)

- Malus domestica Borkh. (Rosaceae) [167]

109. Dysaphis (Dysaphis) emicis (Mimeur, 1935)

- Aneilema sp. (Commelinaceae) [2]

- Melanoseris cyanea Edgew. (=Lactuca hastata DC.) (Asteraceae) [168]

- Rumex nepalensis Spreng. (Polygonaceae) [17, 169, 170]

- Rumex sp. (Polygonaceae) [17]

- Solanum torvum Sw. (Solanaceae) [14]

110. Dysaphis (Dysaphis) foeniculus (Theobald, 1923)

- Foeniculum vulgare Mill. (Apiaceae) [14, 105]

- Fragaria sp. (Rosaceae) [17] 
- Polygonum sp. (Polygonaceae) [167]

- Rumex nepalensis Spreng. (Polygonaceae) [17, 23]

- Rumex sp. (Polygonaceae) [167]

111. Dysaphis (Dysaphis) hirsutissima (Börner, 1940)

= Macrosiphoniella hirsutissima Börner, 1940 [50]

- Artemisia vulgaris L. (Asteraceae) [50]

112. Dysaphis (Dysaphis) longipilosa (Mordvilko, 1928)

- Undet.: unidentified plants [17]

113. Dysaphis (Dysaphis) ramani Das \& Raychaudhuri, 1983

- Crataegus sp. (Rosaceae) [167]

114. Dysaphis (Dysaphis) rumecicola (Hori, 1927)

- Rumex nepalensis Spreng. (Polygonaceae) [87, 167]

- Rumex sp. (Polygonaceae) [167]

115. Dysaphis (Dysaphis) selinumi Chakrabarti \& Medda, 1993

- Selinum wallichianum (DC.) Raizada \& H.O. Saxena (=Selinum tenuifolium Salisb.) (Apiaceae) [167]

116. Dysaphis (Dysaphis) tulipae (Boyer de Fonscolombe, 1841)

- Gladiolus sp. (Iridaceae) [2, 167]

117. Dysaphis (Pomaphis) multisetosa Basu, 1969

=Dysaphis (Dysaphis) multisetosa Basu, 1969

- Pyrus communis L. (Rosaceae) [20, 171, 172]

118. Dysaphis (Pomaphis) pavlovskyana indica Chakrabarti \& Medda, 1993

- Sorbus cuspidata (Spach) Hedl. (Rosaceae) [167]

- Saussurea piptathera Edgew. (Asteraceae) [167]

119. Dysaphis (Pomaphis) plantaginea (Passerini, 1860)

- Plantago sp. [167]

120. Dysaphis (Pomaphis) pyri (Boyer de Fonscolombe, 1841)

= Sappaphis pyri (Boyer de Fonscolombe, 1841) [1]

- Malus domestica Borkh. (=Pyrus malus L.) (Rosaceae) [106, 165]

- Prunus persica (L.) Batsch [167]

- Sorbus khasiana (Decne.) Rehder (= Pyrus khasiana Hooker.f.) (Rosaceae) [20]

- Pyrus pashia Buch.-Ham. ex D. Don (Rosaceae) [173]

121. Dysaphis sp.

- Brassica oleracea L. (Brassicaceae) [14]

- Carduus sp. (Asteraceae) [88]

- Cnicus wallichii Hook.f. (Asteraceae) [17, 129]

- Cotoneaster bacillaris Wall. ex Lindl. (Rosaceae) [14]

- Crataegus sp. (Rosaceae) [88]

- Fragaria nilgerrensis Schltdl. ex J. Gay (Rosaceae) 14]

- Fragaria sp. (Rosaceae) [14]

- Laggera pterodonta (DC.) Benth. (Asteraceae) [14]

- Lyonia ovalifolia (Wall.) Drude (=Pieris ovalifolis (Wall. D. Don)) (Ericaceae) [14]

- Nicotiana sp. (Solanaceae) [14]

- Peucedanum tenuifolium Thunb. (Apiaceae) [14]

- Torilis japonica (Hoatt.) DC. (Apiaceae) [167]

- Viburnum sp. (Adoxaceae) [14]

International J. Research Studies in Zoology (IJRSZ) 


\section{CONCLUSION}

Food plants of 25 genera and 107 species of aphids belonging to the tribe Macrosiphini (Acutosiphon to Dysaphis) are catalogued in this contribution. These aphids feeds on 397 plant species belonging to 79 families. Most suffered families are Asteraceae ( 81 species) followed by Rosaceae (35 species), Fabaceae (27 species), Brassicaceae (22 species), Lamiaceae (18 species) and Solanaceae (17 species). Among them, several species are crops of agricultural and horticultural importance.

\section{REFERENCES}

[1] Raychaudhuri, D.N. (Ed.), Food Plant Catalogue of Indian Aphididae. Graphic Printall, Culcutta (India). pp. 204 (1983).

[2] Chakrabarti, S. and Sarkar, A., A supplement to the food-plant catalogue of Indian Aphididae. J. Aphidol. 15, 9-62 (2001).

[3] Singh, G. and Singh, R., Distribution and economic importance of Aphis (Aphis) craccivora Koch (Aphidini, Aphidinae, Aphididae, Hemiptera) and its food plants in India. Internat. J. Recent Adv. Multidisc. Res. 3(12), (2016)

[4] Singh, G. and Singh, R., Distribution of Aphis (Aphis) spiraecola Patch 1914 (Aphidini, Aphidinae, Aphididae, Hemiptera) and its food plants recorded in India. Internat. J. Recent Adv. Multidisc. Res. 3(12), 2100-2111, (2016).

[5] Singh, G. and Singh, R., Food plant records of aphids (Aphididae, Sternorrhyncha, Hemiptera) in India belonging to subfamilies Aiceoninae, Anoeciinae, Chaitophorinae and Drepanosiphinae. Internat. J. Zool. Investig. 2(2), 281-295, (2016).

[6] Singh, G. and Singh, R., Food plant records of Aphidini (Aphidinae, Aphididae, Hemiptera) in India. J. Ent. Zool. Stud. 5(1), (2017).

[7] Tiwari, A.K. and Singh, R., Effect of host plants on the morphology of green peach aphid, Myzus persicae (Sulzer) (Homoptera, Aphididae), Internat. J. Zool. Investig. 2(1), 133-146 (2016).

[8] Heie, O.E. and Wegierek, P., Diagnoses of the higher taxa of Aphidomorpha (Hemiptera, Sternorrhyncha). Redia 92, 261-269 (2009).

[9] Remaudiere, G. and Remaudiere, M., Catalogue of the World's Aphididae. Instut National de la Recherche Agronomique, Paris, pp. 473 (1997).

[10] Nieto Nafría, J.M. and Favret, C. (Eds.), Registers of Family-Group and Genus-Group Taxa of Aphidoidea (Hemiptera Sternorrhyncha), Universidad de León. León (Spain), pp. 466 (2011).

[11] Favret, C., Aphid Species File. Version 5.0/5.0. <http,//Aphid.SpeciesFile.org> (accessed on January 2, 2017).

[12] Chan, C.K., Forbes, A.R. and Raworth, D.A., Aphid transmitted viruses and the vectors of the world. Agriculture Canada, Ottawa, ON, Tech. Bull. 1991-3E, pp. 216 (1991).

[13] Ghosh, L.K. and Basu, R.C., Insecta, Hemiptera, Aphididae. In Fauna of West Bengal. State Fauna Series, Zool. Surv. India 3, 125-318 (1997).

[14] Chakrabarti, S., Aphids of north western India with special reference to Kumaon range, Uttar Pradesh, Ph. D. thesis. University of Calcutta, India, pp. 435 (1972).

[15] Chakrabarti, S., Ghosh, A.K. and Raychaudhuri, D.N., On some undescribed morphs and new records of aphids (Homoptera, Aphididae) from Kumaon Himalaya, India. Curr. Sci. 41, 70-71 (1972).

[16] Chakrabarti, S. and Raychaudhuri, D.N., Aphids from Sundadhirga Valley Kumaon Himalaya, India. Orient. Ins. 9, 195-211 (1975).

[17] Ghosh, L.K., A study on the aphids (Homoptera, Aphididae) of Himachal Pradesh in NorthWest Himalaya, India, Ph. D. thesis, University of Calcutta, India, 360 pp. (1977).

[18] Ghosh, M.R., Basu, R.C. and Raychaudhuri, D.N., Studies on aphids (Homoptera, Aphididae) from eastern India. XXVIII. Acutosiphon, Akkaia and Vesiculaphis. Orient. Ins. 10, 267-276 (1976).

[19] Raychaudhuri, D.N., Taxonomy of the aphids of the Eastern Himalayas. US Pl 480 Project Tech. Rep., pp. 107 (1973). 
[20] Raychaudhuri, D.N. (Ed.), Aphids of North-East India and Bhutan. Zool. Soc., Calcutta, pp. 521 (1980).

[21] Maity, S.P. and Chakrabarti, S., Aphids (Homoptera, Aphididae) of northwest India. III. Records of new aphids from Garhwal Himalaya. Sci. \& Cult. 45, 160-162 (1979).

[22] Basu, R.C., Ghosh, A.K. and Raychaudhuri, D.N., Studies on the aphids (Homoptera: Aphididae) from eastern India. 4. A new genus and records of some sexual froms from Assam. Proc. zool. Soc. Calcutta 23, 83-91 (1970).

[23] Ghosh, L.K., On a collection of aphids (Homoptera, Aphididae) from Himachal Pradesh, India. Orient. Ins. 6, 169-178 (1972).

[24] Chakrabarti, S. and Raychaudhuri, D.N., Aphids (Homoptera: Aphididae) from Sundardhunga Valley, Kumaon Himalaya, India. Orient. Ins. 9, 195-211 (1975).

[25] Ghosh, A.K., Banerjee, H. and Raychaudhuri, D.N., Aphids (Homoptera, Aphididae) collected from Kameng district, NEFA. Gaveshana 3, 10 (1971).

[26] Raychaudhuri, D.N., Ghosh, M.R. and Basu, R.C., Studies on the aphids (Homoptera, Aphididae) from eastern India. 27. The genera Acyrthosiphon Mordvilko, Metopolophium Mordvilko and Rhodobium Hille Ris Lambers. Proc. zool. Soc., Calcutta 28, 115-131 (1975).

[27] Verma, K.D. and Singh, H.V., Sophora tementosa - A new host for Macrosiphum (Acyrthosiphon) gossypii Mordv. (Homoptera: Aphididae). Curr. Sci. 44, 368 (1975).

[28] Behura, B.K., Aphids of India. Survey of published information. Recent Adv. Zool. India 1961, 25-78 (1963).

[29] David, S.K., Notes on South Indian Aphids. Indian J. Ent. 18, 1-9 (1956).

[30] Ghosh, A.K., Chakrabarti, S., Chowdhuri, A.N. and Raychaudhuri, D.N., Aphids (Homoptera) of Himachal Pradesh, India. Orient. Ins. 3, 327-334 (1969).

[31] Kar, I., Basu, G. and Khuda-Bukhsh, A.R., A Check-list of chromosomes in aphids (Homoptera: Aphididae) worked out in India along with the names and families of their host plants. Environ. and Ecol. 8(1), 414-428 (1990).

[32] David, S.K. and Ghorpade, K.D., Two species of aphids (Homoptera, Aphididae) new to India and four others new to southern India. Orient. Ins. 8, 195-198 (1974).

[33] Banerjee, H., Ghosh, A.K. and Raychaudhuri, D.N., On a collection of aphids (Homoptera, Aphididae) from Kullu valley, west Himalaya. Orient. Ins. 3, 255-264 (1969).

[34] Basu, R.C., Ghosh, A.K. and Raychaudhuri, D.N., A new species of Eutrichosiphum and notes on other new records of aphids (Insecta, Homoptera) from NEFA (Arunachal), Sci. \& Cult. 38, 494-495 (1972).

[35] Basu, A.N., Some aphids new to India with description of a new subspecies. Curr. Sci. 30, 390391 (1961a).

[36] Behura, B.K., Suppliment to aphids of India - a survey of published information. Prakruti - J. Utkal Univ. Sci. 3, 40-65 (1965).

[37] Ghosh, L.K., A Conspectus of Aphididae (Homoptera) of Himachal Pradesh in North-west Himalaya, India. Zool. Surv. India (Tech. Monog. No. 16, pp.1-282 (1986).

[38] Ghosh, L.K., Aphids of NEFA, India (Homoptera: Aphididae), Sci. \& Cult. 36, 562-563 (1970).

[39] Bhalla, O.P. and Pawar, A.D., A survey of insect and non-insect pests of economic importance in Himachal Pradesh. Published by Department of Entomology and Zoology, College of Agriculture, Chambaghat, Solan (H.P.) (1980).

[40] Bindra, O.S. and Sekhon, S.S., Additional new records of aphids from the Kangra, Kulu and Lahaul valleys (India). Bull. Ent. 10, 156-157 (1969).

[41] David, S.K. and Hameed, S.F., One new species and two new records of aphids (Homoptera, Aphididae) from Lahaul in northwestern Himalaya. Orient. Ins. 9, 213-219 (1975).

[42] Chakrabarti, S. Ghosh, A.K. and Raychaudhuri, D.N., Three new species of aphids (Homoptera: Aphididae) from northeast Himalayas, India. Kontyu 39, 368-372 (1971).

[43] Krishnamurthi, B., Aphididae of Mysore. III. Indian J. Ent. 10, 51-53 (1948).

[44] Despande, V.G., A preliminary account of the Aphididae of Poona. J. Bombay nat. Hist. Soc. 39, 740-744 (1938). 
[45] Ghulam-Ullah, Studies of Indian Aphididae - I, The Aphid fauna of Delhi. Indian J. Ent. 2, 13 25 (1940).

[46] David, S.K., A new genus and three new species of aphids from India. Indian J. Ent. 20, 175180 (1958).

[47] David, S.K., Aphids capable of infesting potato in India and their relationship to the crop in South India. J. South Indian Hort. 6, 67-74 (1958).

[48] Joshi, S. and Poorani, J., Aphids of Karnataka. http,//www.aphidweb.com (accessed on 2 January, 2017).

[49] Ghosh, L.K., On a collection of aphids (Homoptera, Aphididae) from Uttar Pradesh, India. Sci. \& Cult. 35, 493-494 (1969).

[50] Rao, V.P., Survey for natural enemies in India. CIBC. Indian Station, U.S. PL 480 Project, Final Tech. Rep. pp. 1-93 (1969).

[51] Verma, K.D., Additional records of Jammu and Kashmir aphids. Sci. \& Cult. 37, 248-249 (1971).

[52] Ahmad, M.E. and Singh, R., Records of Macrosiphini of northeastern Uttar Pradesh and its relationship with food plants and natural enemies. J. Aphidol. 9, 80-86 (1995).

[53] Singh, R. Upadhyay, B.S. Singh, D. Chaudhary, H.C., Aphids (Homoptera, Aphididae) and their parasitoids in north-eastern Uttar Pradesh. J. Aphidol. 13, 49-62 (1999).

[54] Raychaudhuri, D.N. Poddar, S.C. and Raychaudhuri, D., Study of the genus Aphidius (Hymenoptera, Aphidiidae) of India. Entomon 7, 11-22 (1982).

[55] Raychaudhuri, D., Taxonomy and biology of aphids (Homoptera, Aphididae) of Manipur. Ph. D. thesis, University of Calcutta, India, pp. 308+XVI (1978).

[56] Agarwala, B.K. and Raychaudhuri, D.N., Parasites and predastors of aphids in Sikkim and Manipur (northeast India). III. Entomon 5, 39-42 (1980).

[57] Agarwala, B.K. Raychaudhuri, D. and Raychaudhuri, D.N., Parasites and predators of aphids in Sikkim and Manipur (Northeast india). III. Entomon 5, 39-42 (1980).

[58] Agarwala, B.K. and Raychaudhuri, D.N., Parasites and predators of aphids (Homoptera, Aphididae) in northeast India. IV. Twelve coleopteran and two dipteran predators of aphids from Sikkim. Entomon 6, 207-209 (1981).

[59] Basu, A.N. and Banerjee, S.N., Aphids of economic plants of West Bengal. Indian Agric. 2, 89112 (1958).

[60] Joshi, H.C. and Mathur, Y.K., Aphids of Rajasthan. Madras Agric. J. 54, 239-243 (1967).

[61] Basu, A.N., New genera and species of aphids from the Darjeeling district, India (Homoptera, Aphididae). J. Linn. Soc. Zool. 45, 223-243 (1964).

[62] Chakrabarti, S. and Raychaudhuri, D.N., Callipterine aphids (Homoptera: Aphididae) of northeast India and Bhutan. Proc. zool. Soc. Calcutta 28, 71-101 (1975).

[63] Verma, K.D., A new genus, some species, a subspecies and some new records of aphids from N.W. India. Sci. \& Cult. 35, 28-29 (1969).

[64] Chakrabarti, S. Ghosh, A.K. and Raychaudhuri, D.N., New records of aphids (Insecta: Homoptera) from Uttar Pradesh, India. Sci. \& Cult. 37, 247-248 (1971).

[65] David, S.K. Narayanan, K. and Rajasingh, S.G., A new genus and four new species of aphids (Homoptera) from India. Oriental Ins. 5, 557-570 (1971).

[66] Ghosh, A.K. Banerjee. H. and Raycnaudhuti, D.N., Aphid (Homoptera: Insecta) species collected from Manipur and Nagaland. Gaveshana 3, 60-61 (1971).

[67] Raychaudhuri, D.N. Ghosh, L.K. and Das, S.K., Studies on the aphids (Homoptera, Aphididae) from north and northwest India-I. Ins. Matsum. n. s. 20, 1-42 (1980).

[68] Ghosh, A.K., A list of aphids (Homoptera, Aphididae) from India and adjacent countries. J. Bombay nat. Hist. Soc. 71, 201-225 (1974).

[69] Stroyan, H.L.G. and Nagaich, B.B.. A new subspecies of Acrythosipon Mordvilko 1904 (Homoptera : Aphididae) from Rubus ellipticus in north India. Proc. R. ent. Soc. Lond. (B), 33 : 59-62 (1964).

[70] Basu, R.C. and Raychaudhuri, D.N., A study on the sexuales of aphids (Homoptera, Apididae) in India. Rec. Zool. Surv. India, Occ. Paper 18, 1-54 (1980). 
[71] Bhagat R.C., Aphids (Insecta) of agricultural importance in J\&K state, India: a checklist and biodiversity. Internat. J. Food, Agric. \& Vet. Sci. 2(3), 116-125 (2012).

[72] Bhagat, R.C., New records and hosts of aphid parasitoids (Hymenoptera: Aphidiidae) from Kashmir, India. J. Bombay nat. Hist. Soc. 81, 93-98 (1984).

[73] Chakrabarti, S. Ghosh, A.K. and Raychaudhuri, D.N., New Records of Aphids (Insecta: Homoptera) from Uttar Pradesh, India. Sci. \& Cult. 37, 247-248 (1971).

[74] Das, B.C. and Chakrabarti, S., Aphidiid parasitoids (Hymenoptera: Aphidiidae) of graminaceous aphids in Garhwal of Western Himalayas. Orient. Ins. 24, 265-372 (1989).

[75] Chakrabarti, S. Sarkar, S. and Das, B.C., Aphid parasitoids (Hymenoptera: Braconidae: Aphidiinae) from western Himalaya. J. Aphidol. 16, 45-49 (2002).

[76] Medda, P.K. Sarkar, A. and Chakrabarti, S., Willow infesting aphids (Homoptera: Aphididae) of India and adjacent countries. J. Aphidol. 11(1), 83-97 (1997).

[77] Sathe, T.V. and Jadhav, B.V., Indian Pest Aphids. Daya Publishing House, New Delhi, pp. 211 (2008).

[78] Agarwala, B.K., Some aspects of aphid (Homoptera, Insecta) studies in Sikkim and Bhutan Ph. D. thesis, University of Calcutta, India, pp. 383 (1979).

[79] Ghosh, M.R., Ghosh, A.K., and Raychaudhuri, D.N., Studies on aphids (Homoptera, Aphididae) from eastern India. Proc. zool. Soc. Calcutta, 24, 163-168 (1971).

[80] David, S.K., Some rare aphids in new regions in India. J. Bombay nat. Hist. Soc. 66, 323-326 (1969).

[81] Raychaudhuri, D.N., Ghosh, D., Raychaudhuri, D. and Agarwala, B.K., Studies on the aphids (Homoptera: Aphididae) from south India. I. Ins. Matsum. n. s. 23, 1-20 (1981).

[82] Ghosh, A.K., Banerjee, H. and Raychaudhuri, D.N., Studies on the aphids (Homoptera: Aphididae) from eastern India. V. New species, new records on sexuals of some species of aphids from NEFA and Assam. Orient. Ins. 5, 103-110 (1971).

[83] Agarwala, B.K. and Raychaudhuri, D.N., Two new species of aphids (Homoptera, Aphididae) from Sikkim, northeast India. Entomon 2, 77-80 (1977).

[84] Hille Ris Lambers, D. and Basu, A.N., Some new or little known genera, subgenera, species and subspecies of Aphididae from India (Homoptera, Aphididae), Ent. Bericht. 26, 12-20 (1966).

[85] Maity, S.P., Bhattacharya, D.K. and Chakrabarti, S., Four new species of aphids (Homoptera: Aphididae) from Garhwal Himalaya, Uttar Pradesh, India. Ann. Zool. 36(26), 501-516 (1982).

[86] David, S.K. Rajasingh, S.G. and Narayanan, K., New genus, new species and new morphs of aphids (Homoptera) from India. Orient. Ins. 6, 35-44 (1972).

[87] Bhagat, R.C., New records of the aphids (Homoptera, Aphididae) from Kashmir (India). Sci. \& Cult. 47, 134-136 (1981).

[88] Rishi, N.D. (1975), Abst. Symp. 'Recent Trends in Aphidological Studies', Bhubaneswar, p. 52.

[89] Ghosh, A.K., Fern infesting aphids (Homoptera: Aphididae) in India. Indian. J. Hort. 31, 104 109 (1974).

[90] Ghosh, A.K., Floral assemblage and faunal diversity in Aphidoidea (Homoptera: Aphididae) in Eastern India. Bull. zool. Surv. India 2, 171-176 (1980).

[91] Reddi, S.G.E. Kumai, A. and Lal, B., First report of the aphid, Amphorophora ampullata (Homoptera, Aphididae) on the fern, Hypolepis polipodioides (Hypolepidaceae) from western Himalayas (India). Amer. Fern J. 103(3), 185-187 (2013).

[92] Dharmadhikari, P.R. and Ramaseshiah, G., Recent records of aphidiids (Hym. Aphidiidae) in India. CIBC Tech. Bull. No. 13, 83-89 (1970).

[93] Ghosh, A.K. and Raychaudhuri, D.N., Studies on the aphids (Homoptera, Aphididae) from eastern India. XVI. A new subgenus, Anaulacorthum. XXXX. Seven new species and notes on little known species from northeast India and Sikkim. Proc. zool. Soc. Calcutta, 25, 93-107 (1972).

[94] David, S.K. Sekhon, S.S. and Bindra, O.S., New aphids from northeast India (Homoptera, Aphididae). Bull. Ent. 11, 150-155 (1970). 
[95] Bindra, O.S. and Sekhon, S.S., New records of aphids from Punajb and Kulu valley. Bull. Ent. 10, 103-104 (1969).

[96] Verma, K.D., Additions to the aphid fauna of north west India. Sci. \& Cult. 31: 389 (1965).

[97] Verma, K.D., a new aphid genus from northwest India (Homoptera, Aphididae), Indian J. Ent. 28, 507 -509 (1966).

[98] Bhagat, R.C., On aphids (Homoptera, Aphididae) and their parasitoids (Aphidiidae, Hymenoptera) infesting forest trees in Kashmir valley, India. Indian Forester, 111, 467-474 (1985).

[99] Chakrabarti, S. and Medda, P.K., Taxonomic studies on some aphids (Homoptera, Aphididae) from India. Orient. Ins. 23, 133-141 (1989).

[100] Chakrabarti, S. and Maity, S.P., A new genus and four new species of aphids (Homoptera: Aphididae) from Northwest Himalaya, India. Orient. Ins. 18, 195-212 (1984).

[101] Ghosh, A.K. Basu, R.C. and Raychaudhuri, D.N., Studies on the aphids (Homoptera,Aphididae) from eastern India. Orient. Ins. 4, 65-76 (1970).

[102] Agarwala, B.K. Pramanik, D.R. and Raychaudhuri D., Some new and little known macrosiphine aphids (Homoptera: Aphididae) from India. Akitu, N.S. 47, 1-7 (1982).

[103] Ghosh, M.R. Ghosh, A.K. and Raychaudhuri, D.N., Studies on the aphids (Homoptera, Aphididae) from eastern India. III. New genus, new species and new records from north Bengal and Sikkim. Orient. Ins. 4, 377-393 (1970).

[104] Kumar, R. and Burkhardt, C.C., A new species of genus Xenosiphonaphis with four siphunculi and two new species of subgenus Aulacorthum (Homoptera, Aphididae). J. Kansas Ent. Soc. 44, 172-181 (1971).

[105] Chakrabarti, S. Ghosh, A.K. and Raychaudhuri, D.N., A new genus, a new species and further records of aphids (Homoptera, Aphididae) from the Kumaon hills, northwest Himalaya, India. Orient. Ins. 6, 387-400 (1972b).

[106] Raha, S.K., Studies on the aphids (Homoptera, Insecta) of Nagaland, Ph. D. thesis, University of Calcutta, India, pp. 212 (1979).

[107] Basu, A.N., Further records of new and little known aphids (Homoptera) from West Bengal, India. Orient. Ins. 3, 355-371 (1969).

[108] Raha, S.K. Singh, T.K., Raychaudhuri, D. and Raychaudhuri, D.N., New records of aphids (Homoptera, Aphididae) from Manipur and Nagaland. Sci. \& Cult. 43, 452-453 (1977).

[109] Ghosh, A.K. and Raychaudhuri, D.N., Further records of aphids (Homoptera, Insecta) from NEFA. Sci. \& Cult. 37, 483-484 (1971).

[110] Chakrabarti, S. Chowdhuri, A.N. and Raychaudhuri, D.N., Further records of aphids (Homoptera, Aphididae) from Himachal Pradesh, India. Sci. \& Cult. 40, 461-462 (1974).

[111] Stary, P. and Bhagat, R.C., Records and descriptions of aphid parasitoids from Kashmir, India (Hymenoptera, Aphidiidae). Acta Ent. Bohem. 75, 387-393 (1978).

[112] Lefroy, H.M and Howlett, F.M., Indian Insect Life. A manual of the insects of the plains (Tropical India), W. Thacker and Co. London, pp. $743-748$ (1909).

[113] George, C.J., South Indian Aphididae. J. Asiatic. Soc. Bengal, (N.S.) 23, 1-12 (1927).

[114] Krishnamurthi, B., Aphididae of Mysore. II. J. Bombay nat. Hist. Soc. 34, 411-419 (1931).

[115] Lal, K.B. and Siddiqui, Z.A., Biology of the peach leaf curling aphid on the Kumaun hills. Indian J. Ent. 14, 191-196 (1952).

[116] Sharma, P.L. and Bhalla. O.P., A survey of insect pests of economic importance in Himachal Pradesh. Indian J. Ent. 26, 318-331 (1964).

[117] Ghosh, D. Debnath, N. and Chakrabarti, S., Predators and parasites of aphids (Homoptera, Aphididae) from northwest Himalaya, ten species of syrphids (Diptera, Syrphidae) from Garhwal range. Entomon 10, 301-303 (1985).

[118] Raychaudhuri, D.N. Dutta, S. Agarwala, B.K. Raha, S.K. and Raychaudhuri, D., Some parasites and predators of aphids from northeast India and Bhutan. II. Entomon 4, 163-166 (1979).

[119] Ghosh, D. Debnath, N. and Chakrabarti, S., Predators and parasites of aphids from northwest and western Himalaya. IV. Twelve species of heteropterans (Heteroptera: Insecta) from Garhwal and Kumaon ranges. Proc. zool. Soc. Calcutta 39, 15-19 (1986). 
[120] Chakrabarti, S. and Banerjee, P.K., Host-alternation in Aphids from Western and Northwest Himalaya, India. Proceedings of Critical Issues in Aphid Biology, pp. 43-53 (1993).

[121] David, S.K., Notes on South Indian Aphids. IV. Aphidinae (continued). Indian J. Ent. 19, 289299 (1957).

[122] Ghosh, A.K. and Agarwala, B.K., A catalogue of aphidiid (Hymenoptera: Aphidiidae) parasites of aphids (Homoptera) of India. J. Bombay nat. Hist. Soc. 79, 125-134 (1985).

[123] Das, B.C. and Chakrabarti, S., Seasonal occurrence of Aphidius matricariae Haliday (Aphidiidae, Hymenoptera) in Garhwal range of northwest Himalaya. Indian J. Ent. 50, 388389 (1988).

[124] Chakrabarti, S., Diversity and biosystematics of gall-inducing aphids (Homoptera, Aphididae) and their galls in the Himalaya. Orient. Ins. 41, 35-54 (2007).

[125] Takada, H. and Rishi, N.D., Records of fifteen species of Aphidiide (Hymenoptera) from Kashmir, India with descriptions of three new species. Kontyu 48, 234-240(1980).

[126] Ghosh, L.K. and Verma, K.D., A new host record of Brachycaudus helichrysi (Kaltenbach) in India (Homoptera: Aphididae). J. Aphidol. 2, 66-68 (1988).

[127] Nayak, M.R.C., Basu, M. and Raychaudhuri, D.N., Parasites and predators of aphids (Homoptera: Aphididae ) from India. Pranikee 3, 7-14 (1982).

[128] Chowdhuri, A.N. Basu, R.C. Chakrabarti, S. and Raychaudhuri, D.N., A list of aphids from Simla including some new and a few others hitherto not known from India. Sci. \& Cult. 34, 133-134 (1968).

[129] Chowdhuri, A.N. Basu, R.C. and Raychaudhuri, D.N., A new species of Cavariella del Guercio and other newly recorded aphids (Homoptera, Aphididae) from Simla, Himachal Pradesh. Sci. \& Cult. 35, 334 (1969).

[130] Chowdhuri, A.N. Ghosh, A.K. Banerjee, H. and Raychaudhuri, D.N., A few sexual forms and seven newly recorded species of aphids (Aphididae, Homoptera) from Simla. Sci. \& Cult. 36, 550 (1970).

[131] Agarwala, B.K. Ghosh, D. Das, S.K. Poddar, S.C. and Raychaudhuri, D.N., Parasites and predators of aphids (Homoptera, Aphididae) from India - V. New records of two aphidiid parasites, nine arachnid and one dipteran predators from India. Entomon 6, 233-238 (1981).

[132] Basu, R.C. Ghosh, A.K. and Raychaudhuri, D.N., Studies on the aphids (Homoptera, Aphididae) from eastern India. 18. Five new species and thirty new records from Assam. Proc. zool. Soc. Calcutta, 26, 89-101 (1973).

[133] Ghosh, L.K., A note on the preliminary survey of aphids (Homoptewra) from Bihar, India. Sci. \& Cult. 36, 419-420 (1970).

[134] Ghosh, L.K. Raychaudhuri, D. Raychaudhuri, D.N. and Raha, S.K., An account of the genus Brevicoryne Goot (Homoptera, Aphididae) in India with description of sexual morph of Brevicoryne barbarae Nevsky. Bull. zool. Surv. India, 3, 63-68 (1980).

[135] Chakrabarti, S., Chaitophorinae (Homoptera, Aphididae) of India with descriptions of three new species from northwest India. Orient. Ins. 11, $205-224$ (1977).

[136] Basu, R.C. and Raychaudhuri, D.N., Studies on the aphids (Homoptera, Aphididae) from eastern India. XXXI. The genus Capitophorus. Orient. Ins. 10, 567-572 (1976).

[137] Banerjee, P.K., Chakrabarti, S. and Chakrabarti, S. (1991), Host association and undescribed alate viviparous female of Matsumuraja capitophoroides Hille Ris Lambers (Homoptera: Aphididae). J. Bombay Nat. Hist. Soc. 88, 293-296.

[138] Saha, Santanu and Chakrabarti, S., New records of aphids (Homoptera: Aphididae) from Garhwal range of Western Himalaya, India. J. Bom. nat. Hist. Soc. 85, 633-635 (1988).

[139] David, S.K., Addition to the aphid fauna of India. J. Bombay nat. Hist. Soc. 53, 479-482 (1956).

[140] Chakrabarti, S. and Maity, S.P., Aphids (Homoptera, Aphididae) of northwest India. II. Two new species and an undescribed morph. Ann. Zool. 35, 93-104 (1980).

[141] Bhattacharya, D.K. and Chakrabarti, S., Stratigraphic distribution and host association of Aphidinae aphids (Homoptera, Aphididae) of Garhwal range of Western Himalaya. J. Aphidol. 1, 64-69 (1987). 
[142] Raychaudhuri, D. Singh, T.K. and Raychaudhuri, D.N., Some new and unknown aphids (Homoptera, Aphididae) from Manipur, northeast India. Entomon 9(1), 53-56 (1984).

[143] Ghosh, A.K. and Raychaudhuri, D.N., New aphids from northeast India (Homoptera, Aphididae). Ann. Ent. Soc. Amer. 61, 752-755 (1968).

[144] Ghosh, L.K., Description of alate viviparous female of Capitophorus hippophaes indica Ghosh and Raychaudhuri (Homoptera, Aphididae). Bull. Ent. 11 (1), 55-56 (1970).

[145] Ghosh, L.K., Additions to the aphid fauna of Bihar with the first record of an aphid sexuale (Homoptera, Aphididae). J. Bomb. nat. Hist. Soc. 73, 416-418 (1977).

[146] Chakrabarti, S., Two new species of aphids (Homoptera: Aphididae) from northwest India. Zool. J. Linn. Soc. 58, 263-271 (1976).

[147] Ghosh, A.K. and Raychaudhuri, D.N., Studies on the aphids (Homoptera, Aphididae) from eastern India. XIII. New species and further new records from Assam. Orient. Ins. 6, 371-386 (1972).

[148] Maity, S.P. Bhattacharya, D.K. and Chakrabarti, S., Aphids (Homoptera: Aphididae) of northwest India. V. New records of aphids from Garhwal Himalaya. Sci. \& Cult. 46, 311-312 (1980).

[149] Ghosh, D., Medda, P.K. and Chakrabarti, S., Holocycly, seasonal activity, morphometry and natural enemies of willow aphid, Cavariella (Cavariella) aegopodii (Scopoli) (Homoptera: Aphididae) in the Indian region. Proc. Indian Acad. Sci. 95(2), 181-186 (1986).

[150] Basu, R.C., Ghosh, A.K. and Raychaudhuri, D.N., Studies on the aphids (Homoptera, Aphididae) from eastern India. VIII. A new genus and records of aphids from Assam. Sci. \& Cult. 40, 41-43 (1974).

[151] Ghosh, A.K. Ghosh, M.R. and Raychaudhuri, D.N., Studies on the aphids (Homoptera: Aphididae) from eastern India. VII. New species and new records from West Bengal. Orient. Ins. 5, 209-222 (1971).

[152] David, S.K. and Rajasingh, S.G., New records of aphids (Insecta, Homoptera) from Assam, India. Proc. zool. Soc. Calcutta, 22, 151-157 (1969).

[153] Chakrabarti, S. and Medda, P.K., A review of the genus Ceruraphis (Homoptera: Aphididae). Orient. Ins. 19, 125-132 (1985).

[154] Bhattacharya, D.K., Maity, S.P. and Chakrabarti, S., Aphids (Homoptera, Aphididae) of northwest India. VII. Hitherto unknown morphs of three species described from India and Pakistan. Entomon 5, 335-339 (1980).

[155] Chakrabarti, S. Mandal, A.K. and Raha, S., Rhododendron infesting aphids (Homoptera: Aphididae) of the Himalayas. Zool. J. Linn. Soc. 78, 349-362 (1983).

[156] David, S.K. Rajasingh, S.G. and Narayanan, K., The myzaphidines (Homoptera) of India with description of three new species. Orient. Ins. 4, 395-406 (1970).

[157] Chakrabarti, S. Ghosh, A.K. and Chowdhuri, A.N., Aphids (Homoptera, Aphididae) of Himachal Pradesh, India. Orient. Ins. 4, 447-452 (1970).

[158] Chakrabarti, S., A new synonymy and hitherto unknown morphs of Chaetosiphon Mordvilko (Insecta, Homoptera, Aphididae) described from India. Sci. \& Cult. 42, 335-336 (1976).

[159] Chakrabarti, S. and Bhattacharya, D.K., New genus and species of aphids (Homoptera, Aphididae) from north-western Himalaya. Ann. Zool. 36(28), 539-547 (1982).

[160] Verma, K.D., A new species and two undescribed morphs of aphids (Homoptera, Aphididae) from north-west India. Indian J. Ent. 36, 200-202 (1974).

[161] Blackman R.L. and Eastop V.F., Aphids on the World's Herbaceous Plants and Shrubs, 2 Volume Set, John Wiley and Sons, pp. 1460 (2008).

[162] Rao, S.N. and Kulkarni, P.P., Studies on the aphid fauna (Homoptera, Aphididae) of Marathwada (Maharastra), II. Marathwada Univ. J. Nat. Sci. 16, 141-150 (1977).

[163] Bhattacharya, D.K. Mandal, A.K. and Chakrabarti, S., New and hitherto not known species of aphids (Homoptera: Aphididae) producing leaf galls in the north west Himalayas, India. Entomon, 8, 13-17 (1983). 
[164] Bhattacharya, D.K. and Dey, S.R., A new species and a new record of Aphids (Homoptera: Aphididae) from Garhwal range of Western Himalaya, India. Entomon, 21, 285-289(1996).

[165] Raha, S.K. and Raychaudhuri, D.N., Studies on the aphids (Homoptera, Aphididae) of Nagaland. Entomon, 6, 317-323 (1981).

[166] Bhattacharya, D.K., Aphids (Homoptera: Aphididae) infesting Artemisia spp. plants of western and northwest Himalaya. J. Aphidol. 5, 68-74 (1991).

[167] Chakrabarti, S. and Medda, P.K., Taxonomic studies on Dysaphis Börner (Homoptera : Aphididae) from the Indian subcontinent. Orient. Ins. 27, 93-130 (1993).

[168] Ghosh, A.K., Basu, R.C. and Raychaudhuri, D.N., A new genus and seven new species of aphids (Homoptera) from India. Orient. Ins. 3, 245-254 (1969).

[169] Ghosh, A.K., Notes on two new records of aphids (Homoptera : Aphididae) from India. Bull. Ent. 10, 107-109 (1969).

[170] Ghosh, L.K., Notes on two new records of aphids (Homoptera : Aphididae) from India. Bull. Ent. 10, 107-109 (1969).

[171] Ghosh, M.R. and Raychaudhuri, D.N., Aphids (Homoptera: Aphididae) infesting rosaceous fruit plants in Darjeeling district of West Bengal and Sikkim. Entomon 6, 61-68 (1981).

[172] Basu, A.N., Four new genera and nine species of aphids (Homoptera : Aphididae) from West Bengal, India. Orient. Ins. 3, 169-186 (1969).

[173] Ghosh, A.K., New species and new records of aphids (Homoptera : Aphididae) from Northeast India. Orient. Ins. 8, 161-175 (1974).

\section{AUTHORS' BIOGRAPHY}

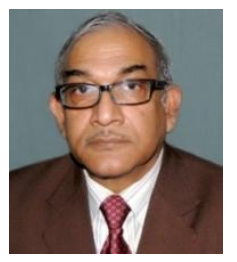

Dr. Rajendra Singh, is Professor and Head of the Department of Zoology, Deen Dayal Upadhyaya University of Gorakhpur and has 40 years of experience in the fields of bioecology and biosystematics of aphids and their parasitoids and predators, published more than 200 research articles of international repute, authored 6 text/reference books, supervised one D.Sc. and $25 \mathrm{Ph}$. D. students and completed 12 research projects. He is fellow/life member of several scientific organizations/ societies, Chief Editor of J. Aphidol. and is on the editorial/reviewer panel of several International Journals. Prof. Singh also served in the university as Pro-Vice Chancellor.

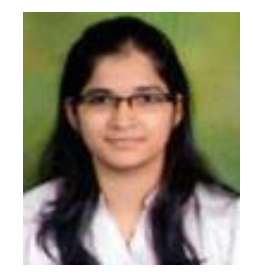

Ms. Garima Singh, is a Ph.D. Student in the Department of Zoology, Rajasthan University, Jaipur and doing research work on the factors affecting progeny sex ratio of an aphid parasitoid, Lipolexis oregmae (Gahan) (Hymenoptera: Braconidae, Aphidiinae). She has published three review articles. She has compiled the food plants of black bean aphid, Aphis craccivora Koch, cotton aphid, Aphis gossypii Glover, green peach aphid, Myzus persicae (Sulzer) and spirea aphid, Aphis spiraecola Patch recorded in India. 